\title{
Semigroup Methods for the M/G/1 Queueing Model with Working Vacation and Vacation Interruption
}

\author{
Ehmet Kasim ${ }^{1}$ \\ ${ }^{1}$ College of Mathematics and Systems Science, Xinjiang University, Urumqi 830046, P.R.China \\ Correspondence: Ehmet Kasim, College of Mathematics and Systems Science, Xinjiang University, Urumqi 830046, \\ P.R.China. E-mail: ehmetkasim@163.com
}

Received: June 30, 2016 Accepted: July 25, 2016 Online Published: September 26, 2016

doi:10.5539/jmr.v8n5p56 URL: http://dx.doi.org/10.5539/jmr.v8n5p56

\begin{abstract}
By using the strong continuous semigroup theory of linear operators we prove that the M/G/1 queueing model with working vacation and vacation interruption has a unique positive time-dependent solution which satisfies probability conditions. When the both service completion rate in a working vacation period and in a regular busy period are constant, by investigating the spectral properties of an operator corresponding to the model we obtain that the time-dependent solution of the model strongly converges to its steady-state solution.
\end{abstract}

Keywords: M/G/1 queueing model with working vacation and vacation interruption, $C_{0}-$ semigroup, dispersive operator, resolvent set, eigenvalue

\section{Introduction}

According to (Zhang \& Hou, 2010), the M/G/1 queueing system with working vacation and vacation interruption can be described by the following system of partial differential equations:

$$
\begin{aligned}
& \frac{d p_{0,0}(t)}{d t}=-\lambda p_{0,0}(t)+\int_{0}^{\infty} \mu_{0}(x) p_{1,0}(x, t) d x+\int_{0}^{\infty} \mu_{1}(x) p_{1,1}(x, t) d x, \\
& \frac{\partial p_{1,0}(x, t)}{\partial t}+\frac{\partial p_{1,0}(x, t)}{\partial x}=-\left[\lambda+\theta+\mu_{0}(x)\right] p_{1,0}(x, t), \\
& \frac{\partial p_{n, 0}(x, t)}{\partial t}+\frac{\partial p_{n, 0}(x, t)}{\partial x}=-\left[\lambda+\theta+\mu_{0}(x)\right] p_{n, 0}(x, t)+\lambda p_{n-1,0}(x, t), \quad \forall n \geq 2, \\
& \frac{\partial p_{1,1}(x, t)}{\partial t}+\frac{\partial p_{1,1}(x, t)}{\partial x}=-\left[\lambda+\mu_{1}(x)\right] p_{1,1}(x, t), \\
& \frac{\partial p_{n, 1}(x, t)}{\partial t}+\frac{\partial p_{n, 1}(x, t)}{\partial x}=-\left[\lambda+\mu_{1}(x)\right] p_{n, 1}(x, t)+\lambda p_{n-1,1}(x, t), \forall n \geq 2,
\end{aligned}
$$

with boundary conditions:

$$
\begin{aligned}
p_{1,0}(0, t)= & \lambda p_{0,0}(t), \\
p_{n, 0}(0, t)= & 0, \quad \forall n \geq 2, \\
p_{n, 1}(0, t)= & \theta \int_{0}^{\infty} p_{n, 0}(x, t) d x+\int_{0}^{\infty} \mu_{0}(x) p_{n+1,0}(x, t) d x \\
& +\int_{0}^{\infty} \mu_{1}(x) p_{n+1,1}(x, t) d x, \forall n \geq 1,
\end{aligned}
$$

and initial condition:

$$
p_{0,0}(0)=1, p_{0,1}(0)=0, p_{m, 0}(x, 0)=p_{m, 1}(x, 0)=0, \quad \forall m \geq 1 .
$$

Where, $(x, t) \in[0, \infty) \times[0, \infty) ; p_{0,0}(t)$ represents the probability that there is no customer in the system and the server is in a working vacation period at time $t ; p_{n, 0}(x, t) d x(n \geq 1)$ is the probability that at time $t$ the server is in a working vacation period and there are $n$ customers in the system with elapsed service time of the customer undergoing service lying in $(x, x+d x] ; p_{n, 1}(x, t) d x(n \geq 1)$ is the probability that at time $t$ the server is in a regular busy period and there are $n$ customers in the system with elapsed service time of the customer undergoing service lying in $(x, x+d x] ; \lambda$ is the mean arrival rate of customers; $\theta$ is the vacation duration rate of the server; $\mu_{0}(x)$ is the service rate of the server while the server is in a working vacation period and satisfies

$$
\mu_{0}(x) \geq 0, \quad \int_{0}^{\infty} \mu_{0}(x) d x=\infty .
$$


$\mu_{1}(x)$ is the service rate of the server while the server is in a regular busy period and satisfying

$$
\mu_{1}(x) \geq 0, \quad \int_{0}^{\infty} \mu_{1}(x) d x=\infty .
$$

Queuing situations in which the idle server may take vacations encounter in computer, communication and manufacturing systems, etc. In a classical vacation queue, a server may completely stop the service or do some additional work during a vacation. Proposing various vacation policies provides more flexibility for optimal design and operation control of the system. Therefore, many researchers studied such queueing system, see (Doshi, 1986; Takagi, 1990; Madan, 1992; Gupur, 2002; Gupur, 2010; Gupur \& Guo, 2002; Lu \& Gupur, 2010), for instance.

In many real life congestion situations, the server can be utilized for ancillary work and a different rate during the vacation period. Such a queueing situation is called queue with working vacation. (Servi \& Finn, 2002) first studied the M/M/1 queueing system with multiple working vacation and obtained the transform formulae for the distribution of the number of customers in the system and the sojourn time in a steady state. Since then, Queueing models with working vacation have been studied by several researchers, see (Jain \& Agrawal, 2007; Kim, Choi \& Chae, 2003; Li, Tian, \& Ma, 2007). Moreover, (Wu \& Takagi, 2006) extended Servi and Finn's (Servi \& Finn, 2002) M/M/1 queueing system to an M/G/1 queueing system with multiple working vacation, where the service times during regular service period and working vacation period. (Zhang \& Hou, 2010) considered the $\mathrm{M} / \mathrm{G} / 1$ queueing system with working vacation and vacation interruption where the server enters into vacations when there are no customers and it can take service at a lower rate during the vacation period. If there are customers in the system at the instant of a service completion during the vacation period, the server will come back to the normal working level no matter whether or not the vacation has ended. otherwise, it continues the vacation. Firstly, by using supplementary variable technique they established the above model and gave the Laplace-Stieltjes transform of the stationary waiting time. Then, they obtain the queue length distribution and service status at an arbitrary epoch in steady state condition under following hypothesis:

$$
\lim _{t \rightarrow \infty} p_{0,0}(t)=p_{0,0}, \quad \lim _{t \rightarrow \infty} p_{n, 0}(x, t)=p_{n, 0}(x), \quad \lim _{t \rightarrow \infty} p_{n, 1}(x, t)=p_{n, 1}(x), \quad n \geq 1
$$

In addition, they also perform some numerical examples to study the effect of various parameters on the system's characteristics. By reading the paper we have found that the above hypothesis implies the following two hypotheses:

Hypothesis 1 The model has a unique time-dependent solution.

Hypothesis 2 The time-dependent solution converges to its steady-state solution.

So far, any results about this model have not been found in the literature. In this paper, we do dynamic analysis for the queueing model by using the idea of (Gupur, Li, \& Zhu, 2001), that is, we investigate above two hypotheses. First of all, we convert the model into an abstract Cauchy problem by choosing a suitable Banach space as a state space and introducing an operator corresponding to the model and its domain. By using the Hille-Yosida theorem and Phillips theorem as well as Fattorini theorem we prove that the model has a unique positive time-dependent solutions and therefore we obtain that the Hypothesis 1 is hold. Next, when the both service rate in a working vacation period and service rate in a regular busy period are constant, we study the asymptotic behavior of its time-dependent solution, i.e., we study the Hypothesis 2. Firstly, we determine the expression of the adjoint operator of the operator corresponding to the model and deduce the resolvent set of the operator. Then, we verify that 0 is eigenvalue of the underlying operator and its adjoint operator with geometric multiplicity one. Thus, by using Theorem 14 in (Gupur, Li, \& Zhu, 2001) we obtain that the time-dependent solution of the model strongly converges to its steady-state solution.

For simplicity, we introduce some notations as follows:

$$
\begin{array}{cc}
\Gamma_{1}=\left(\begin{array}{ccccc}
\lambda & 0 & 0 & 0 & \ldots \\
0 & 0 & 0 & 0 & \ldots \\
0 & 0 & 0 & 0 & \ldots \\
\vdots & \vdots & \vdots & \vdots & \ddots
\end{array}\right) \quad \Gamma_{2}=\left(\begin{array}{ccccc}
0 & \theta & 0 & 0 & \ldots \\
0 & 0 & \theta & 0 & \ldots \\
0 & 0 & 0 & \theta & \ldots \\
\vdots & \vdots & \vdots & \vdots & \ddots
\end{array}\right) \\
\Gamma_{3}=\left(\begin{array}{cccccc}
0 & 0 & \mu_{0}(x) & 0 & 0 & \cdots \\
0 & 0 & 0 & \mu_{0}(x) & 0 & \cdots \\
0 & 0 & 0 & 0 & \mu_{0}(x) & \ldots \\
\vdots & \vdots & \vdots & \vdots & \vdots & \ddots
\end{array}\right) \quad \Gamma_{4}=\left(\begin{array}{ccccc}
0 & \mu_{1}(x) & 0 & 0 & \ldots \\
0 & 0 & \mu_{1}(x) & 0 & \ldots \\
0 & 0 & 0 & \mu_{1}(x) & \ldots \\
\vdots & \vdots & \vdots & \vdots & \ddots
\end{array}\right)
\end{array}
$$

Take a state space as follows:

$$
X=\left\{\left(p_{0}, p_{1}\right) \mid p_{0} \in Y_{1}, p_{1} \in Y_{2},\left\|\left(p_{0}, p_{1}\right)\right\|=\left\|p_{0}\right\|_{Y_{1}}+\left\|p_{1}\right\|_{Y_{2}}<\infty\right\},
$$




$$
\begin{aligned}
& Y_{1}=\left\{p_{0} \in \mathbb{R} \times L^{1}[0, \infty) \times \cdots\left|\left\|p_{0}\right\|=\right| p_{0,0} \mid+\sum_{n=1}^{\infty}\left\|p_{n, 0}\right\|_{L^{1}[0, \infty)}<\infty\right\}, \\
& Y_{2}=\left\{p_{1} \in L^{1}[0, \infty) \times L^{1}[0, \infty) \cdots \mid\left\|p_{1}\right\|=\sum_{n=1}^{\infty}\left\|p_{n, 1}\right\|_{L^{1}[0, \infty)}<\infty\right\} .
\end{aligned}
$$

It is obvious that $X$ is a Banach space. Now we define operators and their domain as follows.

$$
\begin{gathered}
\left.A\left(\begin{array}{c}
p_{0,0} \\
p_{1,0}(x) \\
p_{2,0}(x) \\
p_{3,0}(x) \\
\vdots
\end{array}\right),\left(\begin{array}{c}
p_{1,1}(x) \\
p_{2,1}(x) \\
p_{3,1}(x) \\
p_{4,1}(x) \\
\vdots
\end{array}\right)\right)=\left(\left(\begin{array}{ccccc}
-\lambda & 0 & 0 & 0 & \ldots \\
0 & -\frac{d}{d x} & 0 & 0 & \ldots \\
0 & 0 & -\frac{d}{d x} & 0 & \ldots \\
0 & 0 & 0 & -\frac{d}{d x} & \ldots \\
\vdots & \vdots & \vdots & \vdots & \ddots
\end{array}\right)\left(\begin{array}{c}
p_{0,0} \\
p_{1,0}(x) \\
p_{2,0}(x) \\
p_{3,0}(x) \\
\vdots
\end{array}\right),\left(\begin{array}{ccccc}
-\frac{d}{d x} & 0 & 0 & 0 & \ldots \\
0 & -\frac{d}{d x} & 0 & 0 & \ldots \\
0 & 0 & -\frac{d}{d x} & 0 & \ldots \\
0 & 0 & 0 & -\frac{d}{d x} & \ldots \\
\vdots & \vdots & \vdots & \vdots & \ddots
\end{array}\right)\left(\begin{array}{c}
p_{1,1}(x) \\
p_{2,1}(x) \\
p_{3,1}(x) \\
p_{4,1}(x) \\
\vdots
\end{array}\right)\right) \\
D(A)
\end{gathered}
$$

here

$$
\begin{gathered}
\mathcal{D}_{0}=-\left(\lambda+\theta+\mu_{0}(x)\right), \quad \mathcal{D}_{1}=-\left(\lambda+\mu_{1}(x)\right) \\
E\left(\left(\begin{array}{c}
p_{0,0} \\
p_{1,0}(x) \\
p_{2,0}(x) \\
\vdots
\end{array}\right),\left(\begin{array}{c}
p_{1,1}(x) \\
p_{2,1}(x) \\
p_{3,1}(x) \\
\vdots
\end{array}\right)\right)=\left(\left(\begin{array}{c}
\int_{0}^{\infty} \mu_{0}(x) p_{1,0}(x) d x+\int_{0}^{\infty} \mu_{1}(x) p_{1,1}(x) d x \\
0 \\
0 \\
\vdots
\end{array}\right),\left(\begin{array}{l}
0 \\
0 \\
0 \\
\vdots
\end{array}\right)\right), \quad D(E)=X .
\end{gathered}
$$

Then the above system of equations (1.1)-(1.3) can be written as an abstract Cauchy problem in Banach space X.

$$
\left\{\begin{array}{l}
\frac{d\left(p_{0}, p_{1}\right)(t)}{d t}=(A+U+E)\left(p_{0}, p_{1}\right)(t), \quad t \in(0, \infty), \\
\left.\left(p_{0}, p_{1}\right)(0)=\left(\begin{array}{l}
1 \\
0 \\
\vdots
\end{array}\right),\left(\begin{array}{l}
0 \\
0 \\
\vdots
\end{array}\right)\right)
\end{array}\right.
$$

\section{Well-posedness of the System (1.4)}

In this section, in order to obtain well-posedness of the system (1.4), we first need to prove that $A+U+E$ generates a positive contraction $C_{0}$ - semigroup $T(t)$ on $X$.

Theorem 2.1 If $\mu_{0}(x)$ and $\mu_{1}(x)$ are measurable functions and satisfy $\overline{\mu_{0}}=\sup _{x \in[0, \infty)} \mu_{0}(x)<\infty$ and $\overline{\mu_{1}}=\sup _{x \in[0, \infty)} \mu_{1}(x)<\infty$, then $A+U+E$ generates a positive contraction $C_{0}-$ semigroup $T(t)$.

A detailed proof of the Theorem 2.1 can be found in the Appendix. It is not difficult to verify that $X^{*}$, the dual space of $X$, is as follows.

$$
X^{*}=\left\{\begin{array}{l|l}
\left(q_{0}^{*}, q_{1}^{*}\right) & \begin{array}{l}
q_{0}^{*} \in Y_{1}^{*}, \quad q_{1}^{*} \in Y_{2}^{*}, \\
\left\|\left(q_{0}^{*}, q_{1}^{*}\right)\right\| \|=\sup \left\{\left\|q_{0}^{*}\right\|\left\|_{1}^{*},\right\| q_{1}^{*}\|\|_{Y_{2}^{*}}\right\}
\end{array}
\end{array}\right\},
$$


here

$$
\begin{aligned}
& Y_{1}^{*}=\left\{q_{0}^{*} \mid \begin{array}{l}
q_{0}^{*}(x)=\left(q_{0,0}^{*}, q_{1,0}^{*}(x), q_{2,0}^{*}(x), q_{3,0}^{*}(x), \cdots\right), \\
\left\|q_{0}^{*}\right\| \mid=\sup \left\{\left|q_{0,0}^{*}\right|, \sup _{n \geq 1}\left\|q_{n, 0}^{*}\right\|_{L^{\infty}[0, \infty)}\right\}<\infty
\end{array}\right\}, \\
& Y_{2}^{*}=\left\{\begin{array}{l|l}
q_{1}^{*} \mid \begin{array}{l}
q_{1}^{*}(x)=\left(q_{0,1}^{*}, q_{1,1}^{*}(x), q_{2,1}^{*}(x), q_{3,1}^{*}(x), \cdots\right) \\
\left\|q_{1}^{*}\right\|=\sup _{n \geq 1}\left\|q_{n, 1}^{*}\right\|_{L^{\infty}[0, \infty)}<\infty
\end{array}
\end{array}\right\} .
\end{aligned}
$$

It is obvious that $X^{*}$ is a Banach space. If we take a set $S$ in $X$ as

$$
S=\left\{\left(p_{0}, p_{1}\right) \in X \mid p_{0,0} \geq 0, p_{n, 0}(x) \geq 0, p_{n, 1}(x) \geq 0, \forall n \geq 1, x \in[0, \infty)\right\} .
$$

Then $S$ is cone in $X$. for $\left(p_{0}, p_{1}\right) \in D(A) \cap S$, we take

$$
\left(q_{0}^{*}, q_{1}^{*}\right)=\left\|\left(p_{0}, p_{1}\right)\right\|\left(\left(\begin{array}{l}
1 \\
1 \\
1 \\
\vdots
\end{array}\right),\left(\begin{array}{l}
1 \\
1 \\
1 \\
\vdots
\end{array}\right)\right) \in X^{*}
$$

For such $\left(p_{0}^{*}, p_{1}^{*}\right) \in X^{*}$, by using the boundary condition we have

$$
\begin{aligned}
& \left\langle(A+U+E)\left(p_{0}, p_{1}\right),\left(q_{0}^{*}, q_{1}^{*}\right)\right\rangle=\left\|\left(p_{0}, p_{1}\right)\right\|\left\{-\lambda p_{0,0}+\int_{0}^{\infty} \mu_{0}(x) p_{1,0}(x) d x+\int_{0}^{\infty} \mu_{1}(x) p_{1,1}(x) d x\right\} \\
& +\int_{0}^{\infty}\left\|\left(p_{0}, p_{1}\right)\right\|\left\{-\frac{d p_{1,0}(x)}{d x}-\left(\lambda+\theta+\mu_{0}(x)\right) p_{1,0}(x)\right\} d x \\
& +\sum_{n=2}^{\infty} \int_{0}^{\infty}\left\|\left(p_{0}, p_{1}\right)\right\|\left\{-\frac{d p_{n, 0}(x)}{d x}-\left(\lambda+\theta+\mu_{0}(x)\right) p_{n, 0}(x)+\lambda p_{n-1,0}(x)\right\} \\
& +\int_{0}^{\infty}\left\|\left(p_{0}, p_{1}\right)\right\|\left\{-\frac{d p_{1,1}(x)}{d x}-\left(\lambda+\mu_{1}(x)\right) p_{1,1}(x)\right\} d x \\
& +\sum_{n=2}^{\infty} \int_{0}^{\infty}\left\|\left(p_{0}, p_{1}\right)\right\|\left\{-\frac{d p_{n, 1}(x)}{d x}-\left(\lambda+\mu_{1}(x)\right) p_{n, 1}(x)+\lambda p_{n-1,1}(x)\right\} \\
& =\left\|\left(p_{0}, p_{1}\right)\right\|\left\{-\lambda p_{0,0}+\int_{0}^{\infty} \mu_{0}(x) p_{1,0}(x) d x+\int_{0}^{\infty} \mu_{1}(x) p_{1,1}(x) d x\right. \\
& -\sum_{n=1}^{\infty} \int_{0}^{\infty} d p_{n, 0}(x)-\sum_{n=1}^{\infty} \int_{0}^{\infty}\left(\lambda+\theta+\mu_{0}(x)\right) p_{n, 0}(x) d x+\lambda \sum_{n=1}^{\infty} \int_{0}^{\infty} p_{n, 0}(x) d x \\
& \left.-\sum_{n=1}^{\infty} \int_{0}^{\infty} d p_{n, 1}(x)-\sum_{n=1}^{\infty} \int_{0}^{\infty}\left(\lambda+\mu_{1}(x)\right) p_{n, 1}(x) d x+\lambda \sum_{n=1}^{\infty} \int_{0}^{\infty} p_{n, 1}(x) d x\right\} \\
& =\left\|\left(p_{0}, p_{1}\right)\right\|\left\{-\lambda p_{0,0}+\int_{0}^{\infty} \mu_{0}(x) p_{1,0}(x) d x+\int_{0}^{\infty} \mu_{1}(x) p_{1,1}(x) d x\right. \\
& +\sum_{n=1}^{\infty} p_{n, 0}(0)-\sum_{n=1}^{\infty} \int_{0}^{\infty}\left(\theta+\mu_{0}(x)\right) p_{n, 0}(x) d x \\
& \left.+\sum_{n=1}^{\infty} p_{n, 1}(0)-\sum_{n=1}^{\infty} \int_{0}^{\infty} \mu_{1}(x) p_{n, 1}(x) d x\right\} \\
& =\left\|\left(p_{0}, p_{1}\right)\right\|\left\{-\lambda p_{0,0}+\int_{0}^{\infty} \mu_{0}(x) p_{1,0}(x) d x+\int_{0}^{\infty} \mu_{1}(x) p_{1,1}(x) d x\right. \\
& +\lambda p_{0,0}-\sum_{n=1}^{\infty} \int_{0}^{\infty}\left(\theta+\mu_{0}(x)\right) p_{n, 0}(x) d x \\
& +\sum_{n=1}^{\infty} \int_{0}^{\infty} \mu_{1}(x) p_{n+1,1}(x) d x+\theta \sum_{n=1}^{\infty} \int_{0}^{\infty} p_{n, 0}(x) d x
\end{aligned}
$$




$$
\begin{aligned}
& \left.+\sum_{n=1}^{\infty} \int_{0}^{\infty} \mu_{0}(x) p_{n+1,0}(x) d x-\sum_{n=1}^{\infty} \int_{0}^{\infty} \mu_{1}(x) p_{n, 1}(x) d x\right\} \\
= & 0
\end{aligned}
$$

which shows that $A+U+E$ is a conservative operator. Since the initial value $\left.\left(p_{0}, p_{1}\right)(0)\right) \in D\left(A^{2}\right) \cap S$, by using the Fattorini theorem (Fattorini, 1983) we obtain the following result. see (Gupur, Li, \& Zhu, 2001) for detail proof.

Theorem 2.2 $T(t)$ is isometric for the initial value of the system (1.4), that is,

$$
\left\|T(t)\left(p_{0}, p_{1}\right)(0)\right\|=\left\|\left(p_{0}, p_{1}\right)(0)\right\|, \quad t \in[0, \infty) .
$$

From Theorem 2.1 and Theorem 2.2 we obtain well-posedness of the system (1.4).

Theorem 2.3 If $\mu_{0}(x)$ and $\mu_{1}(x)$ are satisfy $\overline{\mu_{0}}=\sup _{x \in[0, \infty)} \mu_{0}(x)<\infty$ and $\overline{\mu_{1}}=\sup _{x \in[0, \infty)} \mu_{1}(x)<\infty$, then the system (1.4) has a unique positive time-dependent solution $\left(p_{0}, p_{1}\right)(x, t)$ satisfying

$$
\left\|\left(p_{0}, p_{1}\right)(\cdot, t)\right\|=1, \quad \forall t \in[0, \infty)
$$

Proof. Since $\left(p_{0}, p_{1}\right)(0) \in D\left(A^{2}\right) \cap S$, From Theorem 2.1 and (Gupur, Li, \& Zhu, 2001) we know that the system (1.4) has a unique positive time-dependent solution $\left(p_{0}, p_{1}\right)(x, t)$ which can be expressed as

$$
\left(p_{0}, p_{1}\right)(x, t)=T(t)\left(p_{0}, p_{1}\right)(0), \quad t \in[0, \infty)
$$

From which together with Theorem 2.2 (i.e., (2.1)) we derive

$$
\left\|\left(p_{0}, p_{1}\right)(., t)\right\|=\left\|T(t)\left(p_{0}, p_{1}\right)(0)\right\|=\left\|\left(p_{0}, p_{1}\right)(0)\right\|=1, \quad t \in[0, \infty) .
$$

(2.2) just reflects the physical meaning of $\left(p_{0}, p_{1}\right)(x, t)$.

3. Asymptotic Behavior of the Time-Dependent Solution of the System (1.4) when $\mu_{0}(x)=\mu_{0}$ and $\mu_{1}(x)=\mu_{1}$

Lemma 3.1 $(A+U+E)^{*}$, the adjoint operator of $A+U+E$, is as follows.

$$
\begin{aligned}
(A+U+E)^{*}\left(q_{0}^{*}, q_{1}^{*}\right) & =(\mathcal{L}+\mathcal{N}+\mathcal{R}+\mathcal{J})\left(q_{0}^{*}, q_{1}^{*}\right), \\
\left(q_{0}^{*}, q_{1}^{*}\right) & \in D(A+U+E)^{*},
\end{aligned}
$$

where

$$
\begin{aligned}
\mathcal{L}\left(q_{0}^{*}, q_{1}^{*}\right)(x)= & \left(\begin{array}{cccc}
-\lambda & 0 & 0 & \cdots \\
0 & \frac{d}{d x}-\left(\lambda+\theta+\mu_{0}\right) & 0 & \cdots \\
0 & 0 & \frac{d}{d x}-\left(\lambda+\theta+\mu_{0}\right) & \cdots \\
\vdots & \vdots & \vdots & \ddots
\end{array}\right)\left(\begin{array}{c}
q_{0,0}^{*} \\
q_{1,0}^{*}(x) \\
q_{2,0}^{*}(x) \\
\vdots
\end{array}\right), \\
& \left(\begin{array}{cccc}
\frac{d}{d x}-\left(\lambda+\mu_{1}\right) & 0 & 0 & \cdots \\
0 & \frac{d}{d x}-\left(\lambda+\mu_{1}\right) & 0 & \cdots \\
0 & 0 & \frac{d}{d x}-\left(\lambda+\mu_{1}\right) & \cdots \\
\vdots & \vdots & \vdots & \ddots
\end{array}\right)\left(\begin{array}{c}
q_{1,1}^{*}(x) \\
q_{2,1}^{*}(x) \\
q_{3,1}^{*}(x) \\
\vdots
\end{array}\right),
\end{aligned}
$$




$$
\begin{aligned}
& \mathcal{N}\left(q_{0}^{*}, q_{1}^{*}\right)(x)=\left(\left(\begin{array}{ccccc}
0 & 0 & 0 & 0 & \cdots \\
0 & 0 & \lambda & 0 & \cdots \\
0 & 0 & 0 & \lambda & \cdots \\
\vdots & \vdots & \vdots & \vdots & \ddots
\end{array}\right)\left(\begin{array}{c}
q_{0,0}^{*} \\
q_{1,0}^{*}(x) \\
q_{2,0}^{*}(x) \\
\vdots
\end{array}\right)+\left(\begin{array}{cccc}
0 & \lambda & 0 & \cdots \\
0 & 0 & 0 & \cdots \\
0 & 0 & 0 & \cdots \\
\vdots & \vdots & \vdots & \ddots
\end{array}\right)\left(\begin{array}{c}
q_{0,0}^{*} \\
q_{1,0}^{*}(0) \\
q_{2,0}^{*}(0) \\
\vdots
\end{array}\right),\right. \\
& \left.\left(\begin{array}{ccccc}
0 & \lambda & 0 & 0 & \cdots \\
0 & 0 & \lambda & 0 & \cdots \\
0 & 0 & 0 & \lambda & \cdots \\
\vdots & \vdots & \vdots & \vdots & \ddots
\end{array}\right)\left(\begin{array}{c}
q_{1,1}^{*}(x) \\
q_{2,1}^{*}(x) \\
q_{3,1}^{*}(x) \\
\vdots
\end{array}\right)\right) \\
& \mathcal{R}\left(q_{0}^{*}, q_{1}^{*}\right)(x)=\left(\left(\begin{array}{cccc}
0 & 0 & 0 & \cdots \\
\mu_{0} & 0 & 0 & \cdots \\
0 & 0 & 0 & \cdots \\
0 & 0 & 0 & \cdots \\
\vdots & \vdots & \vdots & \ddots
\end{array}\right)\left(\begin{array}{c}
q_{0,0}^{*} \\
q_{1,0}^{*}(0) \\
q_{2,0}^{*}(0) \\
q_{3,0}^{*}(0) \\
\vdots
\end{array}\right)+\left(\begin{array}{ccccc}
0 & 0 & 0 & 0 & \cdots \\
0 & 0 & 0 & 0 & \cdots \\
\mu_{0} & 0 & 0 & 0 & \cdots \\
0 & \mu_{0} & 0 & 0 & \cdots \\
\vdots & \vdots & \vdots & \vdots & \ddots
\end{array}\right)\left(\begin{array}{c}
q_{1,1}^{*}(0) \\
q_{2,1}^{*}(0) \\
q_{3,1}^{*}(0) \\
q_{4,1}^{*}(0) \\
\vdots
\end{array}\right),\right. \\
& \left.\left(\begin{array}{ccccc}
0 & 0 & 0 & 0 & \cdots \\
\mu_{1} & 0 & 0 & 0 & \cdots \\
0 & \mu_{1} & 0 & 0 & \cdots \\
0 & 0 & \mu_{1} & 0 & \cdots \\
\vdots & \vdots & \vdots & \vdots & \ddots
\end{array}\right)\left(\begin{array}{c}
q_{1,1}^{*}(0) \\
q_{2,1}^{*}(0) \\
q_{3,1}^{*}(0) \\
q_{4,1}^{*}(0) \\
\vdots
\end{array}\right)\right) \\
& \mathcal{J}\left(q_{0}^{*}, q_{1}^{*}\right)(x)=\left(\left(\begin{array}{cccc}
0 & 0 & 0 & \cdots \\
\theta & 0 & 0 & \cdots \\
0 & \theta & 0 & \cdots \\
0 & 0 & \theta & \cdots \\
\vdots & \vdots & \vdots & \ddots
\end{array}\right)\left(\begin{array}{c}
q_{1,1}^{*}(0) \\
q_{2,1}^{*}(0) \\
q_{3,1}^{*}(0) \\
q_{4,1}^{*}(0) \\
\vdots
\end{array}\right),\left(\begin{array}{cccc}
\mu_{1} & 0 & 0 & \cdots \\
0 & 0 & 0 & \cdots \\
0 & 0 & 0 & \cdots \\
\vdots & \vdots & \vdots & \ddots
\end{array}\right)\left(\begin{array}{c}
q_{0,0}^{*} \\
q_{1,0}^{*}(0) \\
q_{2,0}^{*}(0) \\
\vdots
\end{array}\right)\right), \\
& D\left((A+U+E)^{*}\right)=\left\{\begin{array}{l|l}
\left(q_{0}^{*}, q_{1}^{*}\right) \in X^{*} & \begin{array}{l}
\frac{d q_{0, n}^{*}(x)}{d x} \text { and } \frac{d q_{1, n}^{*}(x)}{d x} \text { exist and } \\
q_{n, 0}^{*}(\infty)=q_{n, 1}^{*}(\infty)=\alpha, \quad n \geq 1
\end{array}
\end{array}\right\},
\end{aligned}
$$

here $\alpha$ in $D\left((A+U+E)^{*}\right)$ is a constant which is irrelevant to $n$.

The proof is easy computaion, and we omit the detail proof.

\section{Lemma 3.2}

$$
\left\{\begin{array}{l|l|l|}
\gamma \in C & \begin{array}{l}
\operatorname{Re} \gamma+\lambda+\theta+\mu_{0}>0, \operatorname{Re} \gamma+\lambda+\mu_{1}>0, \\
\sup \left\{\frac{\lambda}{|\gamma+\lambda|}, \frac{\lambda\left|\gamma+\lambda+\mu_{1}\right|}{\left(\operatorname{Re} \gamma+\lambda+\mu_{1}\right)\left(\left|\gamma+\lambda+\mu_{1}\right|-\mu_{1}\right.},\right. \\
\frac{\lambda \mu_{0}}{|\gamma+\lambda|\left|\gamma+\lambda+\theta+\mu_{0}\right|}+\frac{\lambda \mu_{1}}{|\gamma+\lambda|\left|\gamma+\lambda+\theta+\mu_{0}\right|\left|\gamma+\lambda+\mu_{1}\right|}+\frac{\lambda}{\left|\gamma+\lambda+\theta+\mu_{0}\right|\left|\operatorname{Re} \gamma+\lambda+\mu_{1}\right|}+\frac{\lambda}{\operatorname{Re} \gamma+\lambda+\theta+\mu_{0}}, \\
\left.\frac{\lambda\left(\theta+\mu_{0}\right)\left|\gamma+\lambda+\mu_{1}\right|}{\left|\gamma+\lambda+\theta+\mu_{0}\right|\left(\operatorname{Re} \gamma+\lambda+\mu_{1}\right)\left(\left|\gamma+\lambda+\mu_{1}\right|-\mu_{1}\right)}+\frac{\lambda}{\operatorname{Re} \gamma+\lambda+\theta+\mu_{0}}\right\}<1 .
\end{array}
\end{array}\right\},
$$

belongs to the resolvent set of $(A+U+E)^{*}$, Especially, all points on the imaginary axis except for zero belong to the resolvent set of $(A+U+E)^{*}$ and $A+U+E$.

Proof. For any given $\left(y_{0}^{*}, y_{1}^{*}\right) \in X^{*}$ we consider the equation $[\gamma I-(\mathcal{L}+\mathcal{R}+\mathcal{J})]\left(q_{0}^{*}, q_{1}^{*}\right)=\left(y_{0}^{*}, y_{1}^{*}\right)$. that is,

$$
\begin{aligned}
& (\gamma+\lambda) q_{0,0}^{*}=y_{0,0}^{*}, \\
& \frac{d q_{1,0}^{*}(x)}{d x}=\left(\gamma+\lambda+\theta+\mu_{0}\right) q_{1,0}^{*}(x)-\mu_{0} q_{0,0}^{*}-\theta q_{1,1}^{*}(0)-y_{0,1}^{*}(x), \\
& \frac{d q_{n, 0}^{*}(x)}{d x}=\left(\gamma+\lambda+\theta+\mu_{0}\right) q_{n, 0}^{*}(x)-\mu_{0} q_{n-1,1}^{*}(0)-\theta q_{n, 1}^{*}(0)-y_{n, 0}^{*}(x), \quad n \geq 2, \\
& \frac{d q_{1,1}^{*}(x)}{d x}=\left(\gamma+\lambda+\mu_{1}\right) q_{1,1}^{*}(x)-\mu_{1} q_{0,0}^{*}-y_{1,1}^{*}(x), \\
& \frac{d q_{n, 1}^{*}(x)}{d x}=\left(\gamma+\lambda+\mu_{1}\right) q_{n, 1}^{*}(x)-\mu_{1} q_{n-1,1}^{*}(0)-y_{n, 1}^{*}(x), \quad n \geq 2, \\
& q_{n, 0}^{*}(\infty)=q_{n, 1}^{*}(\infty)=\alpha, \quad n \geq 1 .
\end{aligned}
$$


By solving (3.1)-(3.5) we have

$$
\begin{aligned}
q_{0,0}^{*}= & \frac{1}{\gamma+\lambda} y_{0,0}^{*}, \\
q_{1,0}^{*}(x)= & a_{1}^{*} e^{\left(\gamma+\lambda+\theta+\mu_{0}\right) x}-e^{\left(\gamma+\lambda+\theta+\mu_{0}\right) x} \int_{0}^{x}\left[\mu_{0} q_{0,0}^{*}+\theta q_{1,1}^{*}(0)\right] e^{-\left(\gamma+\lambda+\theta+\mu_{0}\right) \tau} d \tau \\
& -e^{\left(\gamma+\lambda+\theta+\mu_{0}\right) x} \int_{0}^{x} y_{1,0}^{*}(\tau) e^{-\left(\gamma+\lambda+\theta+\mu_{0}\right) \tau} d \tau, \\
q_{n, 0}^{*}(x)= & a_{n}^{*} e^{\left(\gamma+\lambda+\theta+\mu_{0}\right) x}-e^{\left(\gamma+\lambda+\theta+\mu_{0}\right) x} \int_{0}^{x}\left[\mu_{0} q_{n-1,1}^{*}(0)+\theta q_{n, 1}^{*}(0)\right] e^{-\left(\gamma+\lambda+\theta+\mu_{0}\right) \tau} d \tau \\
& -e^{\left(\gamma+\lambda+\theta+\mu_{0}\right) x} \int_{0}^{x} y_{n, 0}^{*}(\tau) e^{-\left(\gamma+\lambda+\theta+\mu_{0}\right) \tau} d \tau, \quad n \geq 2, \\
q_{1,1}^{*}(x)= & b_{1}^{*} e^{\left(\gamma+\lambda+\mu_{1}\right) x}-e^{\left(\gamma+\lambda+\mu_{1}\right) x} \int_{0}^{x} \mu_{1} q_{0,0}^{*} e^{-\left(\gamma+\lambda+\mu_{1}\right) \tau} d \tau \\
& -e^{\left(\gamma+\lambda+\mu_{1}\right) x} \int_{0}^{x} y_{1,1}^{*}(\tau) e^{-\left(\gamma+\lambda+\mu_{1}\right) \tau} d \tau, \\
q_{n, 1}^{*}(x)= & b_{n}^{*} e^{\left(\gamma+\lambda+\mu_{1}\right) x}-e^{\left(\gamma+\lambda+\mu_{1}\right) x} \int_{0}^{x} \mu_{1} q_{n-1,1}^{*}(0) e^{-\left(\gamma+\lambda+\mu_{1}\right) \tau} d \tau \\
& -e^{\left(\gamma+\lambda+\mu_{1}\right) x} \int_{0}^{x} y_{n, 1}^{*}(\tau) e^{-\left(\gamma+\lambda+\mu_{1}\right) \tau} d \tau, \quad n \geq 2,
\end{aligned}
$$

Multiplying the both side of (3.8), (3.9) by $e^{-\left(\gamma+\lambda+\theta+\mu_{0}\right) x}$, the both side of (3.10), (3.11) by $e^{-\left(\gamma+\lambda+\mu_{1}\right) x}$, and taking the limit $x \rightarrow \infty$ as well as using (3.6) it gives

$$
\begin{aligned}
& a_{1}^{*}=\int_{0}^{\infty}\left[\mu_{0} q_{0,0}^{*}+\theta q_{1,1}^{*}(0)\right] e^{-\left(\gamma+\lambda+\theta+\mu_{0}\right) \tau} d \tau+\int_{0}^{\infty} y_{1,0}^{*}(\tau) e^{-\left(\gamma+\lambda+\theta+\mu_{0}\right) \tau} d \tau, \\
& a_{n}^{*}=\int_{0}^{\infty}\left[\mu_{0} q_{n-1,1}^{*}(0)+\theta q_{n, 1}^{*}(0)\right] e^{-\left(\gamma+\lambda+\theta+\mu_{0}\right) \tau} d \tau+\int_{0}^{\infty} y_{n, 0}^{*}(\tau) e^{-\left(\gamma+\lambda+\theta+\mu_{0}\right) \tau} d \tau, \quad n \geq 2, \\
& b_{1}^{*}=\int_{0}^{\infty} \mu_{1} q_{0,0}^{*} e^{-\left(\gamma+\lambda+\mu_{1}\right) \tau} d \tau+\int_{0}^{\infty} y_{1,1}^{*}(\tau) e^{-\left(\gamma+\lambda+\mu_{1}\right) \tau} d \tau, \\
& b_{n}^{*}=\int_{0}^{\infty} \mu_{1} q_{n-1,1}^{*}(0) e^{-\left(\gamma+\lambda+\mu_{1}\right) \tau} d \tau+\int_{0}^{\infty} y_{n, 1}^{*}(\tau) e^{-\left(\gamma+\lambda+\mu_{1}\right) \tau} d \tau, \quad n \geq 2,
\end{aligned}
$$

By inserting (3.12)-(3.15) into (3.8)-(3.11) we deduce (without lose of generality assume $\operatorname{Re} \gamma+\lambda+\theta+\mu_{0}>0, \operatorname{Re} \gamma+\lambda+\mu_{1}>$ 0)

$$
\begin{aligned}
& q_{1,0}^{*}(x)=\frac{1}{\gamma+\lambda+\theta+\mu_{0}}\left[\mu_{0} q_{0,0}^{*}+\theta q_{1,1}^{*}(0)\right]+e^{\left(\gamma+\lambda+\theta+\mu_{0}\right) x} \int_{x}^{\infty} y_{1,0}^{*}(\tau) e^{-\left(\gamma+\lambda+\theta+\mu_{0}\right) \tau} d \tau, \\
& q_{n, 0}^{*}(x)=\frac{1}{\gamma+\lambda+\theta+\mu_{0}}\left[\mu_{0} q_{n-1,1}^{*}(0)+\theta q_{n, 1}^{*}(0)\right]+e^{\left(\gamma+\lambda+\theta+\mu_{0}\right) x} \int_{x}^{\infty} y_{n, 0}^{*}(\tau) e^{-\left(\gamma+\lambda+\theta+\mu_{0}\right) \tau} d \tau, \quad n \geq 2, \\
& q_{1,1}^{*}(x)=\frac{\mu_{1}}{\gamma+\lambda+\mu_{1}} q_{0,0}^{*}+e^{\left(\gamma+\lambda+\mu_{1}\right) x} \int_{x}^{\infty} y_{1,1}^{*}(\tau) e^{-\left(\gamma+\lambda+\mu_{1}\right) \tau} d \tau, \\
& q_{n, 1}^{*}(x)=\frac{\mu_{1}}{\gamma+\lambda+\mu_{1}} q_{n-1,1}^{*}(0)+e^{\left(\gamma+\lambda+\mu_{1}\right) x} \int_{x}^{\infty} y_{n, 1}^{*}(\tau) e^{-\left(\gamma+\lambda+\mu_{1}\right) \tau} d \tau, \quad n \geq 2,
\end{aligned}
$$

By using (3.18), (3.19) repeatedly we obtain that, by induction,

$$
\begin{aligned}
q_{k, 1}^{*}(x)= & \left(\frac{\mu_{1}}{\gamma+\lambda+\mu_{1}}\right)^{k} q_{0,0}^{*}+\left(\frac{\mu_{1}}{\gamma+\lambda+\mu_{1}}\right)^{k-1} e^{\left(\gamma+\lambda+\mu_{1}\right) x} \int_{0}^{\infty} y_{1,1}^{*}(\tau) e^{-\left(\gamma+\lambda+\mu_{1}\right) \tau} d \tau \\
& +\left(\frac{\mu_{1}}{\gamma+\lambda+\mu_{1}}\right)^{k-2} e^{\left(\gamma+\lambda+\mu_{1}\right) x} \int_{0}^{\infty} y_{2,1}^{*}(\tau) e^{-\left(\gamma+\lambda+\mu_{1}\right) \tau} d \tau+\cdots \\
& +\frac{\mu_{1}}{\gamma+\lambda+\mu_{1}} e^{\left(\gamma+\lambda+\mu_{1}\right) x} \int_{0}^{\infty} y_{k-1,1}^{*}(\tau) e^{-\left(\gamma+\lambda+\mu_{1}\right) \tau} d \tau \\
& +e^{\left(\gamma+\lambda+\mu_{1}\right) x} \int_{x}^{\infty} y_{k, 1}^{*}(\tau) e^{-\left(\gamma+\lambda+\mu_{1}\right) \tau} d \tau, \quad k \geq 1,
\end{aligned}
$$


From (3.20) and (3.7) we estimate that

$$
\begin{aligned}
\left\|q_{k, 1}^{*}\right\|_{L^{\infty}[0, \infty) \leq} \leq & \frac{1}{|\gamma+\lambda|}\left(\frac{\mu_{1}}{\left|\gamma+\lambda+\mu_{1}\right|}\right)^{k}\left|y_{0,0}^{*}\right|+\frac{1}{\operatorname{Re} \gamma+\lambda+\mu_{1}}\left(\frac{\mu_{1}}{\left|\gamma+\lambda+\mu_{1}\right|}\right)^{k-1}\left\|y_{1,1}^{*}\right\|_{L^{\infty}[0, \infty)} \\
& +\frac{\mu_{1}}{\operatorname{Re} \gamma+\lambda+\mu_{1}}\left(\frac{1}{\left|\gamma+\lambda+\mu_{1}\right|}\right)^{k-2}\left\|y_{2,1}^{*}\right\|_{L^{\infty}[0, \infty)}+\cdots \\
& +\frac{1}{\operatorname{Re} \gamma+\lambda+\mu_{1}} \frac{\mu_{1}}{\left|\gamma+\lambda+\mu_{1}\right|}\left\|y_{k-1,1}^{*}\right\|_{L^{\infty}[0, \infty)}+\frac{1}{\operatorname{Re} \gamma+\lambda+\mu_{1}}\left\|y_{k, 1}^{*}\right\|_{L^{\infty}[0, \infty)} \\
\leq & \left\{\frac{1}{|\gamma+\lambda|}\left(\frac{\mu_{1}}{\left|\gamma+\lambda+\mu_{1}\right|}\right)^{k}+\frac{1}{\operatorname{Re} \gamma+\lambda+\mu_{1}} \sum_{j=0}^{k-1}\left(\frac{\mu_{1}}{\left|\gamma+\lambda+\mu_{1}\right|}\right)^{j}\right\}\left\|\left(y_{0}^{*}, y_{1}^{*}\right)\right\| \\
= & \left\{\frac{1}{|\gamma+\lambda|}\left(\frac{\mu_{1}}{\left|\gamma+\lambda+\mu_{1}\right|}\right)^{k}+\frac{\left|\gamma+\lambda+\mu_{1}\right|}{\left(\operatorname{Re} \gamma+\lambda+\mu_{1}\right)\left(\left|\gamma+\lambda+\mu_{1}\right|-\mu_{1}\right)}\right. \\
& \left.-\frac{\left|\gamma+\lambda+\mu_{1}\right|}{\left(\operatorname{Re} \gamma+\lambda+\mu_{1}\right)\left(\left|\gamma+\lambda+\mu_{1}\right|-\mu_{1}\right)}\left(\frac{\mu_{1}}{\left|\gamma+\lambda+\mu_{1}\right|}\right)^{k-1}\right\}\left\|\left(y_{0}^{*}, y_{1}^{*}\right)\right\| \\
\leq & \left\{\frac{\mu_{1}}{|\gamma+\lambda|\left(\operatorname{Re} \gamma+\lambda+\mu_{1}\right)}\left(\frac{\mu_{1}}{\left|\gamma+\lambda+\mu_{1}\right|}\right)^{k-1}+\frac{\left.+\operatorname{Re} \gamma+\lambda+\mu_{1}\right)\left(\left|\gamma+\lambda+\mu_{1}\right|-\mu_{1}\right)}{\left|\gamma+\lambda+\mu_{1}\right|}\right. \\
& \left.-\frac{\mu_{1}}{\left(\operatorname{Re} \gamma+\lambda+\mu_{1}\right)\left(\left|\gamma+\lambda+\mu_{1}\right|-\mu_{1}\right)}\left(\frac{\mu_{1} \mid}{\left|\gamma+\lambda+\mu_{1}\right|}\right)^{k-1}\right\}\left\|\left(y_{0}^{*}, y_{1}^{*}\right)\right\|, \quad k \geq 1 .
\end{aligned}
$$

Note that the following inequality holds.

$$
|\gamma+\lambda| \geq\left|\gamma+\lambda+\mu_{1}\right|-\mu_{1}
$$

which implies

$$
\begin{aligned}
& \frac{\mu_{1}}{|\gamma+\lambda|\left(\operatorname{Re} \gamma+\lambda+\mu_{1}\right)}\left(\frac{\mu_{1}}{\left|\gamma+\lambda+\mu_{1}\right|}\right)^{k-1}-\frac{\left|\gamma+\lambda+\mu_{1}\right|}{\left(\operatorname{Re} \gamma+\lambda+\mu_{1}\right)\left(\left|\gamma+\lambda+\mu_{1}\right|-\mu_{1}\right)}\left(\frac{\mu_{1}}{\left|\gamma+\lambda+\mu_{1}\right|}\right)^{k-1} \\
\leq & \frac{\mu_{1}}{|\gamma+\lambda|\left(\operatorname{Re} \gamma+\lambda+\mu_{1}\right)}\left(\frac{\mu_{1}}{\left|\gamma+\lambda+\mu_{1}\right|}\right)^{k}-\frac{\left|\gamma+\lambda+\mu_{1}\right|}{\left(\operatorname{Re} \gamma+\lambda+\mu_{1}\right)\left(\left|\gamma+\lambda+\mu_{1}\right|-\mu_{1}\right)}\left(\frac{\mu_{1}}{\left|\gamma+\lambda+\mu_{1}\right|}\right)^{k}
\end{aligned}
$$

From which together with (3.21) it follows that

$$
\begin{aligned}
\sup _{k \geq 1}\left\|q_{k, 1}^{*}\right\|_{L^{\infty}[0, \infty) \leq} \leq & \lim _{k \rightarrow \infty}\left\{\frac{\mu_{1}}{|\gamma+\lambda|\left(\operatorname{Re} \gamma+\lambda+\mu_{1}\right)}\left(\frac{\mu_{1}}{\left|\gamma+\lambda+\mu_{1}\right|}\right)^{k-1}+\frac{\left|\gamma+\lambda+\mu_{1}\right|}{\left(\operatorname{Re} \gamma+\lambda+\mu_{1}\right)\left(\left|\gamma+\lambda+\mu_{1}\right|-\mu_{1}\right.}\right. \\
& \left.-\frac{\left|\gamma+\lambda+\mu_{1}\right|}{\left(\operatorname{Re} \gamma+\lambda+\mu_{1}\right)\left(\left|\gamma+\lambda+\mu_{1}\right|-\mu_{1}\right.}\left(\frac{\mu_{1}}{\left|\gamma+\lambda+\mu_{1}\right|}\right)^{k-1}\right\}\left\|\left(y_{0}^{*}, y_{1}^{*}\right)\right\| \\
\leq & \frac{\left|\gamma+\lambda+\mu_{1}\right|}{\left(\operatorname{Re} \gamma+\lambda+\mu_{1}\right)\left(\left|\gamma+\lambda+\mu_{1}\right|-\mu_{1}\right)}\left\|\left(y_{0}^{*}, y_{1}^{*}\right)\right\|,
\end{aligned}
$$

Substituting (3.20) into (3.17) we conclude similarly that

$$
\begin{aligned}
\left\|q_{k, 0}^{*}\right\|_{L^{\infty}[0, \infty)} \leq & \left\{\frac{\mu_{0}}{\left|\gamma+\lambda+\theta+\mu_{0}\right||\gamma+\lambda|}\left(\frac{\mu_{1}}{\left|\gamma+\lambda+\mu_{1}\right|}\right)^{k-1}\right. \\
& +\frac{\left(\theta+\mu_{0}\right)\left|\gamma+\lambda+\mu_{1}\right|}{\left|\gamma+\lambda+\theta+\mu_{0}\right|\left(\operatorname{Re} \gamma+\lambda+\mu_{1}\right)\left(\left|\gamma+\lambda+\mu_{1}\right|-\mu_{1}\right)} \\
& +\frac{\mu_{0}}{\left|\gamma+\lambda+\theta+\mu_{0}\right|\left(\operatorname{Re} \gamma+\lambda+\mu_{1}\right)\left(\left|\gamma+\lambda+\mu_{1}\right|-\mu_{1}\right)}\left(\frac{\mu_{1}}{\left|\gamma+\lambda+\mu_{1}\right|}\right)^{k-2} \\
& +\frac{\theta}{\left|\gamma+\lambda+\theta+\mu_{0}\right||\gamma+\lambda|}\left(\frac{\mu_{1}}{\gamma+\lambda+\mu_{1}}\right)^{k} \\
& +\frac{\theta}{\left|\gamma+\lambda+\theta+\mu_{0}\right|\left(\operatorname{Re} \gamma+\lambda+\mu_{1}\right)\left(\left|\gamma+\lambda+\mu_{1}\right|-\mu_{1}\right)}\left(\frac{\mu_{1}}{\left|\gamma+\lambda+\mu_{1}\right|}\right)^{k-1} \\
& \left.+\frac{1}{\operatorname{Re} \gamma+\lambda+\theta+\mu_{0}}\right\}\left\|\left(y_{0}^{*}, y_{1}^{*}\right)\right\|_{L^{\infty}[0, \infty)}, \quad k \geq 2 .
\end{aligned}
$$


We can easy to check that the following inequality holds.

$$
\begin{aligned}
& \frac{\mu_{0}}{\left|\gamma+\lambda+\theta+\mu_{0}\right||\gamma+\lambda|}\left(\frac{\mu_{1}}{\left|\gamma+\lambda+\mu_{1}\right|}\right)^{k-1} \\
& +\frac{\mu_{0}}{\left|\gamma+\lambda+\theta+\mu_{0}\right|\left(\operatorname{Re} \gamma+\lambda+\mu_{1}\right)\left(\left|\gamma+\lambda+\mu_{1}\right|-\mu_{1}\right)}\left(\frac{\mu_{1}}{\left|\gamma+\lambda+\mu_{1}\right|}\right)^{k-2} \\
& +\frac{\theta}{\left|\gamma+\lambda+\theta+\mu_{0}\right||\gamma+\lambda|}\left(\frac{\mu_{1}}{\gamma+\lambda+\mu_{1}}\right)^{k} \\
& +\frac{\theta}{\left|\gamma+\lambda+\theta+\mu_{0}\right|\left(\operatorname{Re} \gamma+\lambda+\mu_{1}\right)\left(\left|\gamma+\lambda+\mu_{1}\right|-\mu_{1}\right)}\left(\frac{\mu_{1}}{\left|\gamma+\lambda+\mu_{1}\right|}\right)^{k-1} \\
\leq & \frac{\mu_{0}}{\left|\gamma+\lambda+\theta+\mu_{0}\right||\gamma+\lambda|}\left(\frac{\mu_{1}}{\left|\gamma+\lambda+\mu_{1}\right|}\right)^{k} \\
& +\frac{\mu_{0}}{\left|\gamma+\lambda+\theta+\mu_{0}\right|\left(\operatorname{Re} \gamma+\lambda+\mu_{1}\right)\left(\left|\gamma+\lambda+\mu_{1}\right|-\mu_{1}\right)}\left(\frac{\mu_{1}}{\left|\gamma+\lambda+\mu_{1}\right|}\right)^{k-1} \\
& \left.+\frac{\mu_{1}}{\left|\gamma+\lambda+\theta+\mu_{0}\right||\gamma+\lambda|}\right)^{k+1} \\
& +\frac{\theta+\lambda+\mu_{1}}{\left|\gamma+\lambda+\theta+\mu_{0}\right|\left(\operatorname{Re} \gamma+\lambda+\mu_{1}\right)\left(\left|\gamma+\lambda+\mu_{1}\right|-\mu_{1}\right)}\left(\frac{\mu_{1}}{\left|\gamma+\lambda+\mu_{1}\right|}\right)^{k}, k \geq 2 .
\end{aligned}
$$

From which together with (3.23) we know that

$$
\begin{aligned}
\sup _{k \geq 2}\left\|q_{k, 0}^{*}\right\|_{L^{\infty}[0, \infty) \leq} & \lim _{n \rightarrow \infty}\left\{\frac{\mu_{0}}{\left|\gamma+\lambda+\theta+\mu_{0}\right| \gamma+\lambda \mid}\left(\frac{\mu_{1}}{\left|\gamma+\lambda+\mu_{1}\right|}\right)^{k-1}\right. \\
& +\frac{\left(\theta+\mu_{0}\right)\left|\gamma+\lambda+\mu_{1}\right|}{\left|\gamma+\lambda+\theta+\mu_{0}\right|\left(\operatorname{Re} \gamma+\lambda+\mu_{1}\right)\left(\left|\gamma+\lambda+\mu_{1}\right|-\mu_{1}\right)} \\
& +\frac{\mu_{0}}{\left|\gamma+\lambda+\theta+\mu_{0}\right|\left(\operatorname{Re} \gamma+\lambda+\mu_{1}\right)\left(\left|\gamma+\lambda+\mu_{1}\right|-\mu_{1}\right)}\left(\frac{\mu_{1}}{\left|\gamma+\lambda+\mu_{1}\right|}\right)^{k-2} \\
& +\frac{\theta}{\left|\gamma+\lambda+\theta+\mu_{0}\right||\gamma+\lambda|}\left(\frac{\mu_{1}}{\gamma+\lambda+\mu_{1}}\right)^{k} \\
& +\frac{\theta}{\left|\gamma+\lambda+\theta+\mu_{0}\right|\left(\operatorname{Re} \gamma+\lambda+\mu_{1}\right)\left(\left|\gamma+\lambda+\mu_{1}\right|-\mu_{1}\right)}\left(\frac{\mu_{1}}{\left|\gamma+\lambda+\mu_{1}\right|}\right)^{k-1} \\
& \left.+\frac{1}{\operatorname{Re} \gamma+\lambda+\theta+\mu_{0}}\right\}\left\|\left(y_{0}^{*}, y_{1}^{*}\right)\right\|_{L^{\infty}[0, \infty)} \\
=\{ & \frac{\left(\theta+\mu_{0}\right)\left|\gamma+\lambda+\mu_{1}\right|}{\left|\gamma+\lambda+\theta+\mu_{0}\right|\left(\operatorname{Re} \gamma+\lambda+\mu_{1}\right)\left(\left|\gamma+\lambda+\mu_{1}\right|-\mu_{1}\right)} \\
& \left.+\frac{1}{\operatorname{Re} \gamma+\lambda+\theta+\mu_{0}}\right\}\left\|\left(y_{0}^{*}, y_{1}^{*}\right)\right\|_{L^{\infty}[0, \infty)}
\end{aligned}
$$

Combining (3.7), (3.16), (3.22) with (3.24) we estimate

$$
\begin{aligned}
\left\|\left(q_{0}^{*}, q_{1}^{*}\right)\right\|= & \max \left\{\left|p_{0,0}\right|, \sup _{n \geq 1}\left\|q_{n, 0}^{*}\right\|_{L^{\infty}[0, \infty)}, \sup _{n \geq 1}\left\|q_{n, 1}^{*}\right\|_{L^{\infty}[0, \infty)}\right\} \\
= & \max \left\{\frac{1}{|\gamma+\lambda|}, \frac{\left|\gamma+\lambda+\mu_{1}\right|}{\left(\operatorname{Re} \gamma+\lambda+\mu_{1}\right)\left(\left|\gamma+\lambda+\mu_{1}\right|-\mu_{1}\right)},\right. \\
& \frac{\mu_{0}}{\left|\gamma+\lambda \| \gamma+\lambda+\theta+\mu_{0}\right|}+\frac{\theta}{|\gamma+\lambda|\left|\gamma+\lambda+\theta+\mu_{0} \| \gamma+\lambda+\mu_{1}\right|} \\
& +\frac{1}{\left|\gamma+\lambda+\theta+\mu_{0}\right|\left|\operatorname{Re} \gamma+\lambda+\mu_{1}\right|}+\frac{1}{\operatorname{Re} \gamma+\lambda+\theta+\mu_{0}} \\
& \frac{\left(\theta+\mu_{0}\right)\left|\gamma+\lambda+\mu_{1}\right|}{\left|\gamma+\lambda+\theta+\mu_{0}\right|\left(\operatorname{Re} \gamma+\lambda+\mu_{1}\right)\left(\left|\gamma+\lambda+\mu_{1}\right|-\mu_{1}\right)} \\
& \left.+\frac{1}{\operatorname{Re} \gamma+\lambda+\theta+\mu_{0}}\right\}\left\|\left(y_{0}^{*}, y_{1}^{*}\right)\right\|_{L^{\infty}[0, \infty) .}
\end{aligned}
$$


(3.25) shows that

$$
\begin{aligned}
\left\|(\gamma I-\mathcal{L}-\mathcal{R}-\mathcal{J})^{-1}\right\| \leq & \max \left\{\frac{1}{|\gamma+\lambda|}, \frac{\left|\gamma+\lambda+\mu_{1}\right|}{\left(\operatorname{Re} \gamma+\lambda+\mu_{1}\right)\left(\left|\gamma+\lambda+\mu_{1}\right|-\mu_{1}\right.},\right. \\
& \frac{\mu_{0}}{|\gamma+\lambda|\left|\gamma+\lambda+\theta+\mu_{0}\right|}+\frac{\theta \mu_{1}}{|\gamma+\lambda| \gamma+\lambda+\theta+\mu_{0}|| \gamma+\lambda+\mu_{1} \mid} \\
& +\frac{\theta}{\left|\gamma+\lambda+\theta+\mu_{0}\right|\left|\operatorname{Re} \gamma+\lambda+\mu_{1}\right|}+\frac{1}{\operatorname{Re} \gamma+\lambda+\theta+\mu_{0}} \\
& \frac{\left(\theta+\mu_{0}\right)\left|\gamma+\lambda+\mu_{1}\right|}{\left|\gamma+\lambda+\theta+\mu_{0}\right|\left(\operatorname{Re} \gamma+\lambda+\mu_{1}\right)\left(\left|\gamma+\lambda+\mu_{1}\right|-\mu_{1}\right)} \\
& \left.+\frac{1}{\operatorname{Re} \gamma+\lambda+\theta+\mu_{0}}\right\} .
\end{aligned}
$$

Together with the fact $\|\mathcal{N}\|=\lambda$ we conclude that, when

$$
\begin{aligned}
\left\|(\gamma I-\mathcal{L}-\mathcal{R}-\mathcal{J})^{-1} \mathcal{N}\right\| \leq & \left\|(\gamma I-\mathcal{L}-\mathcal{R}-\mathcal{J})^{-1}|\|\mid \mathcal{N}\|\right. \\
\leq & \max \left\{\frac{\lambda}{|\gamma+\lambda|}, \frac{\lambda\left|\gamma+\lambda+\mu_{1}\right|}{\left(\operatorname{Re} \gamma+\lambda+\mu_{1}\right)\left(\left|\gamma+\lambda+\mu_{1}\right|-\mu_{1}\right.},\right. \\
& \frac{\lambda \mu_{0}}{\left|\gamma+\lambda \| \gamma+\lambda+\theta+\mu_{0}\right|}+\frac{\lambda \mu_{1}}{\left|\gamma+\lambda \| \gamma+\lambda+\theta+\mu_{0}\right| \gamma+\lambda+\mu_{1} \mid} \\
& +\frac{\lambda \theta}{\left|\gamma+\lambda+\theta+\mu_{0}\right|\left|\operatorname{Re} \gamma+\lambda+\mu_{1}\right|}+\frac{\lambda\left(\theta+\mu_{0}\right)\left|\gamma+\lambda+\mu_{1}\right|}{\operatorname{Re} \gamma+\lambda+\theta+\mu_{0}} \\
& \frac{\lambda+\lambda+\theta+\mu_{0} \mid\left(\operatorname{Re} \gamma+\lambda+\mu_{1}\right)\left(\left|\gamma+\lambda+\mu_{1}\right|-\mu_{1}\right)}{\mid \gamma+\lambda} \\
& \left.+\frac{\lambda}{\operatorname{Re} \gamma+\lambda+\theta+\mu_{0}}\right\} \\
& <1,
\end{aligned}
$$

$\left[\left(I-(\gamma I-\mathcal{L}-\mathcal{R}-\mathcal{J})^{-1} \mathcal{N}\right]^{-1}\right.$ exists and is bounded. By noting

$$
(\gamma I-\mathcal{L}-\mathcal{R}-\mathcal{J}-\mathcal{N})^{-1}=\left[\left(I-(\gamma I-\mathcal{L}-\mathcal{R}-\mathcal{J})^{-1} \mathcal{N}\right]^{-1}(\gamma I-\mathcal{L}-\mathcal{R}-\mathcal{J})^{-1}\right.
$$

we know that $(\gamma I-\mathcal{L}-\mathcal{R}-\mathcal{J}-\mathcal{N})^{-1}$ exists and is bounded when (3.27) holds. that is to say, (29) belongs to the resolvent set of $(A+U+E)^{*}$ (see Gupur, Li, \& Zhu, 2001). In particular, if $\gamma=i \omega, \omega \in \mathbb{R} \backslash\{0\}, i^{2}=-1$, then all the $\gamma$ naturally belong to (3.27). In fact, by simple calculation, we have

$$
\begin{aligned}
& \frac{\lambda}{\sqrt{\omega^{2}+(\lambda)^{2}}}<\frac{\lambda}{\lambda}=1, \\
& \frac{\lambda}{\lambda+\mu_{1}} \frac{\sqrt{\omega^{2}+\left(\lambda+\mu_{1}\right)^{2}}}{\sqrt{\omega^{2}+\left(\lambda+\mu_{1}\right)^{2}}-\mu_{1}}<1, \\
& \frac{\lambda \mu_{0}}{\sqrt{\omega^{2}+\lambda^{2}} \sqrt{\omega^{2}+\left(\lambda+\theta+\mu_{0}\right)^{2}}}+\frac{\lambda \theta \mu_{1}}{\sqrt{\omega^{2}+\lambda^{2}} \sqrt{\omega^{2}+\left(\lambda+\theta+\mu_{0}\right)^{2}} \sqrt{\omega^{2}+\left(\lambda+\mu_{1}\right)^{2}}} \\
& +\frac{\lambda \theta}{\sqrt{\omega^{2}+\left(\lambda+\theta+\mu_{0}\right)^{2}}\left(\lambda+\mu_{1}\right)}+\frac{\lambda}{\lambda+\theta+\mu_{0}}<1, \\
& \frac{\lambda\left(\theta+\mu_{0}\right) \sqrt{a^{2}+\left(\lambda+\mu_{1}\right)^{2}}}{\sqrt{\omega^{2}+\left(\lambda+\theta+\mu_{0}\right)^{2}}\left(\lambda+\mu_{1}\right)\left(\sqrt{\omega^{2}+\left(\lambda+\mu_{1}\right)^{2}}-\mu_{1}\right)}+\frac{\lambda}{\lambda+\theta+\mu_{0}}<1 .
\end{aligned}
$$

The above inequalities show that the resolvent set of $(A+U+E)^{*}$ contain all points on the imaginary axis except zero so as $(A+U+E)$.

Lemma 3.3 If $\lambda<\mu_{1}$, then 0 is an eigenvalue of $A+U+E$ with geometric multiplicity one. 
Proof. We consider the equation $(A+U+E)\left(p_{0}, p_{1}\right)=0$, which is equivalent to

$$
\begin{aligned}
\lambda p_{0,0}= & \mu_{0} \int_{0}^{\infty} p_{1,0}(x) d x+\mu_{1} \int_{0}^{\infty} p_{1,1}(x) d x, \\
\frac{d p_{1,0}(x)}{d x}= & -\left(\lambda+\theta+\mu_{0}\right) p_{1,0}(x), \\
\frac{d p_{n, 0}(x)}{d x}= & -\left(\lambda+\theta+\mu_{0}\right) p_{n, 0}(x)+\lambda p_{n-1,0}(x), \quad n \geq 2, \\
\frac{d p_{1,1}(x)}{d x}= & -\left(\lambda+\mu_{1}\right) p_{1,1}(x), \\
\frac{d p_{n, 1}(x)}{d x}= & -\left(\lambda+\mu_{1}\right) p_{n, 1}(x)+\lambda p_{n-1,1}(x), \quad n \geq 2, \\
p_{1,0}(0)= & \lambda p_{0,0}, \\
p_{n, 0}(0)= & 0, \quad n \geq 2, \\
p_{n, 1}(0)= & \theta \int_{0}^{\infty} p_{n, 0}(x) d x+\mu_{0} \int_{0}^{\infty} p_{n+1,0}(x) d x \\
& +\mu_{1} \int_{0}^{\infty} p_{n+1,1}(x) d x, \quad n \geq 1,
\end{aligned}
$$

It is difficult to determine the expression of all $p_{n, 0}$ and $p_{n, 1}$ for $n \geq 1$ and to verify $\left(p_{0}, p_{1}\right) \in D(A+U+E)$. Hence, we use an indirect method. We define the probability generating functions for $|z|<1$

$$
P_{0}(x, z)=\sum_{n=1}^{\infty} p_{n, 0}(x) z^{n}, \quad P_{1}(x, z)=\sum_{n=1}^{\infty} p_{n, 1}(x) z^{n}
$$

then Theorem 2.3 ensures that $P_{0}(x, z)$ and $P_{1}(x, z)$ are well-defined. (3.29) and (3.30) gives

$$
\begin{aligned}
\frac{\partial \sum_{n=1}^{\infty} p_{n, 0}(x) z^{n}}{\partial x} & =-\left(\lambda+\theta+\mu_{0}\right) \sum_{n=1}^{\infty} p_{n, 0}(x) z^{n}+\lambda \sum_{n=2}^{\infty} p_{n-1,0}(x) z^{n} \\
& \Longrightarrow \\
\frac{\partial P_{0}(x, z)}{\partial x} & =-\left(\lambda+\theta+\mu_{0}\right) P_{0}(x, z)+\lambda z P_{0}(x, z) \\
& =\left(\lambda z-\lambda-\theta-\mu_{0}\right) P_{0}(x, z) \\
& \Longrightarrow \\
P_{0}(x, z) & =P_{0}(0, z) e^{\left(\lambda z-\lambda-\theta-\mu_{0}\right) x},
\end{aligned}
$$

(3.31) and (3.32) imply

$$
\begin{aligned}
\frac{\partial \sum_{n=1}^{\infty} p_{n, 1}(x) z^{n}}{\partial x} & =-\left(\lambda+\mu_{1}\right) \sum_{n=1}^{\infty} p_{n, 1}(x) z^{n}+\lambda \sum_{n=2}^{\infty} p_{n-1,1}(x) z^{n} \\
& \Longrightarrow \\
\frac{\partial P_{1}(x, z)}{\partial x} & =-\left(\lambda+\mu_{1}\right) P_{1}(x, z)+\lambda z P_{1}(x, z) \\
& =\left(\lambda z-\lambda-\mu_{1}\right) P_{1}(x, z) \\
& \Longrightarrow \\
P_{1}(x, z) & =P_{1}(0, z) e^{\left(\lambda z-\lambda-\mu_{1}\right) x}
\end{aligned}
$$


From (3.33)-(3.35) and (3.28), we deduce

$$
\begin{aligned}
P_{0}(0, z)= & \lambda z p_{0,0} \\
P_{1}(0, z)= & \sum_{n=1}^{\infty} P_{1}(0) z^{n} \\
= & \theta \int_{0}^{\infty} \sum_{n=1}^{\infty} p_{n, 0}(x) z^{n} d x+\mu_{0} \int_{0}^{\infty} \sum_{n=1}^{\infty} p_{n+1,0}(x) z^{n} d x+\mu_{1} \sum_{n=1}^{\infty} \int_{0}^{\infty} p_{n+1,1}(x) z^{n} d x \\
= & \theta \int_{0}^{\infty} P_{0}(x, z) d x+\mu_{0} \int_{0}^{\infty} \frac{1}{z}\left[\sum_{n=1}^{\infty} p_{n, 0}(x) z^{n}-p_{1,0}(x) z\right] d x \\
& +\mu_{1} \int_{0}^{\infty} \frac{1}{z}\left[\sum_{n=1}^{\infty} p_{n, 1}(x) z^{n}-p_{1,1}(x) z\right] d x \\
= & \theta \int_{0}^{\infty} P_{0}(x, z) d x+\frac{\mu_{0}}{z} \int_{0}^{\infty} P_{0}(x, z) d x-\mu_{0} \int_{0}^{\infty} p_{1,0}(x) d x \\
& +\frac{\mu_{1}}{z} \int_{0}^{\infty} P_{1}(x, z) d x-\mu_{1} \int_{0}^{\infty} p_{1,1}(x) d x \\
= & \theta \int_{0}^{\infty} P_{0}(x, z) d x+\frac{\mu_{0}}{z} \int_{0}^{\infty} P_{0}(x, z) d x+\frac{\mu_{1}}{z} \int_{0}^{\infty} P_{1}(x, z) d x-\lambda p_{0,0},
\end{aligned}
$$

By inserting (3.36), (3.37) and (3.38) into (3.39), we determine

$$
\begin{aligned}
& P_{1}(0, z)=\theta \int_{0}^{\infty} P_{0}(0, z) e^{\left(\lambda z-\lambda-\theta-\mu_{0}\right) x} d x+\frac{\mu_{0}}{z} \int_{0}^{\infty} P_{0}(0, z) e^{\left(\lambda z-\lambda-\theta-\mu_{0}\right) x} d x \\
& +\frac{\mu_{1}}{z} \int_{0}^{\infty} P_{1}(0, z) e^{\left(\lambda z-\lambda-\mu_{1}\right) x} d x-\lambda p_{0,0} \\
& =\theta \int_{0}^{\infty} \lambda z p_{0,0} e^{\left(\lambda z-\lambda-\theta-\mu_{0}\right) x} d x+\frac{\mu_{0}}{z} \int_{0}^{\infty} \lambda z p_{0,0} e^{\left(\lambda z-\lambda-\theta-\mu_{0}\right) x} d x \\
& +\frac{\mu_{1}}{z} \int_{0}^{\infty} P_{1}(0, z) e^{\left(\lambda z-\lambda-\mu_{1}\right) x} d x-\lambda p_{0,0} \\
& =\frac{\theta z}{\lambda z-\lambda-\theta-\mu_{0}} \lambda p_{0,0}+\frac{\mu_{0}}{\lambda z-\lambda-\theta-\mu_{0}} \lambda p_{0,0} \\
& +\frac{\mu_{1}}{z\left(\lambda z-\lambda-\mu_{1}\right)} P_{1}(0, z)-\lambda p_{0,0} \\
& \Longrightarrow \\
& P_{1}(0, z)=\frac{\frac{\theta z+\mu_{0}}{\lambda z-\lambda-\theta-\mu_{0}}-1}{1-\frac{\mu_{1}}{z\left(\lambda z-\lambda-\mu_{1}\right)}} \lambda p_{0,0}
\end{aligned}
$$

By (3.40) and the L'Hospital rule we calculate

$$
\begin{aligned}
\lim _{z \rightarrow 1} P_{1}(0, z) & =\lim _{z \rightarrow 1} \frac{\frac{\theta z+\mu_{0}}{\lambda z-\lambda-\theta-\mu_{0}}-1}{1-\frac{\mu_{1}}{z\left(\lambda z-\lambda-\mu_{1}\right)}} \lambda p_{0,0}=\lim _{z \rightarrow 1} \frac{\frac{\theta\left(\lambda z-\lambda-\theta-\mu_{0}\right)-\lambda\left(\theta z+\mu_{0}\right)}{\left(\lambda z-\lambda-\theta-\mu_{0}\right)^{2}}}{\frac{\mu_{1}\left[\left(\lambda z-\lambda-\mu_{1}\right)+\lambda z\right]}{z^{2}\left(\lambda z-\lambda-\mu_{1}\right)^{2}}} \lambda p_{0,0} \\
& =\frac{\frac{\theta\left(-\theta-\mu_{0}\right)-\lambda\left(\theta+\mu_{0}\right)}{\frac{\left(-\theta-\mu_{0}\right)^{2}}{\mu_{1}+\lambda}} \lambda p_{0,0}}{\mu_{1}}=\frac{(\lambda+\theta) \mu_{1}}{\left(\theta+\mu_{0}\right)\left(\mu_{1}-\lambda\right)} \lambda p_{0,0}<\infty
\end{aligned}
$$


By combining (3.36), (3.37), (3.38) and (3.41), we have

$$
\begin{aligned}
\sum_{n=1}^{\infty} p_{n, 0}(x) & =\lim _{z \rightarrow 1} P_{0}(x, z)=\lim _{z \rightarrow 1} P_{0}(0, z) e^{\left(\lambda z-\lambda-\theta-\mu_{0}\right) x} \\
& =\lambda p_{0,0} e^{-\left(\theta+\mu_{0}\right) x} \\
& \Longrightarrow \\
\sum_{n=1}^{\infty} \int_{0}^{\infty} p_{n, 0}(x) d x & =\frac{\lambda}{\theta+\mu_{0}} p_{0,0}<\infty, \\
\sum_{n=1}^{\infty} p_{n, 1}(x) & =\lim _{z \rightarrow 1} P_{1}(x, z)=\lim _{z \rightarrow 1} P_{1}(0, z) e^{\left(\lambda z-\lambda-\mu_{1}\right) x} \\
& =\frac{(\lambda+\theta) \mu_{1}}{\left(\theta+\mu_{0}\right)\left(\mu_{1}-\lambda\right)} \lambda p_{0,0} e^{-\mu_{1} x} \\
& \Longrightarrow \\
\sum_{n=1}^{\infty} \int_{0}^{\infty} p_{n, 1}(x) d x & =\frac{(\lambda+\theta) \lambda}{\left(\theta+\mu_{0}\right)\left(\mu_{1}-\lambda\right)} p_{0,0}<\infty .
\end{aligned}
$$

(3.42) and (3.43) imply

$$
\left\|\left(p_{0}, p_{1}\right)\right\|=\left\|p_{0}\right\|+\left\|p_{1}\right\|<\infty .
$$

This shows that 0 is an eigenvalue of $A+U+E$. Moreover, by solving (3.29) - (3.31) we have

$$
\begin{aligned}
p_{n, 0}(x)= & e^{-\left(\lambda+\theta+\mu_{0}\right) x} \sum_{k=1}^{n} p_{k, 0}(0) \frac{(\lambda x)^{n-k}}{(n-k) !}, \quad n \geq 1, \\
p_{n, 1}(x)= & e^{-\left(\lambda+\mu_{1}\right) x} \sum_{k=1}^{n} p_{k, 1}(0) \frac{(\lambda x)^{n-k}}{(n-k) !}, \quad n \geq 1, \\
p_{1,0}(0)= & \lambda p_{0,0}, \\
p_{n, 0}(0)= & 0, \quad n \geq 2 \\
p_{n, 1}(0)= & \theta \int_{0}^{\infty} p_{n, 0}(x) d x+\mu_{0} \int_{0}^{\infty} p_{n+1,0}(x) d x \\
& +\mu_{1} \int_{0}^{\infty} p_{n+1,1}(x) d x, \quad n \geq 1
\end{aligned}
$$

From the above, we know that the eigenvectors corresponding to zero span one dimensional linear space, that is to say, the geometric multiplicity of 0 is one.

From Theorem 2.3 and Lemma 3.2 we know that 0 is an eigenvalue of $(A+U+E)^{*}$. Furthermore, we deduce the following result.

Lemma 3.4 If $\lambda<\mu_{1}$, then 0 is an eigenvalue of $(A+U+E)^{*}$ with geometric multiplicity one.

Since Theorem 2.3, Lemma 3.2, Lemma 3.3 and Lemma 3.4 are just the conditions of Theorem 14 in (Gupur, Li, \& Zhu, 2001), we conclude the following result.

Theorem 1 If $\lambda<\mu_{1}$, then the time-dependent solution of the system (1.4) strongly converges to its steady-state solution, that is,

$$
\lim _{t \rightarrow \infty}\left\|\left(p_{0}, p_{1}\right)(\cdot, t)-\beta\left(p_{0}, p_{1}\right)(\cdot)\right\|=0,
$$

here $\left(p_{0}, p_{1}\right)(x)$ is the eigenvector in Lemma 3.3 and $\beta$ is decided by the eigenvector in Lemma 3.4 and initial value $\left(p_{0}, p_{1}\right)(0)$.

\section{Conclusion}

In this paper, we do dynamic analysis for the $\mathrm{M} / \mathrm{G} / 1$ queueing model with single working vacation and vacation interruption by using the functional analysis method. we prove the existence of a unique nonnegative time-dependent solution of the model, and when the service completion rate are constant we obtain that the time-dependent solution of the model strongly converges to its steady-state solution. These results confirmed all two hypotheses which stated in the introduction. 
In addition, from Theorem 3.1 we can prove the time-dependent queueing size at the departure point converges a positive number, the time-dependent queueing length and the time-dependent waiting time also converges to the corresponding steady-state queuing length and steady-state waiting time.

Our studies in this field, see (Kasim \& Gupur, 2011) and (Gupur, 2011; Gupur, 2014) for instance, suggest that there are infinitely many eigenvalues of $A+U+E$ on the left half complex plane, that is to say, it is impossible that the timedependent solution of the system (1.4) exponentially converges to its steady-state solution. Of course, it needs to verify. that is our next research work.

\section{Acknowledgements}

The author would like to express his sincere thanks to the anonymous referees and associated editor for his/her careful reading of the manuscript. The author' research work was supported by the Tian Yuan Special Funds of the National Natural Science Foundation of China (No:11526175), the Natural Science Foundation of Xinjiang University(No:BS130104).

\section{Appendix}

Proof of Theorem 2.1 The proof will be divided into four steps. Let us first prove $(\gamma I-A)^{-1}$ exists and is bounded for some $\gamma$. For any given $\left(y_{0}, y_{1}\right) \in X$, we consider the equation $(\gamma I-A)\left(p_{0}, p_{1}\right)=\left(y_{0}, y_{1}\right)$, that is,

$$
\begin{aligned}
(\gamma+\lambda) p_{0,0} & =y_{0,0}, \\
\frac{d p_{n, j}(x)}{d x} & =-\gamma p_{n, j}(x)+y_{n, j}(x), \quad n \geq 1, \quad j=0,1, \\
p_{1,0}(0) & =\lambda p_{0,0}, \quad p_{n, 0}(0)=0, \quad n \geq 2, \\
p_{n, 1}(0) & =\theta \int_{0}^{\infty} p_{n, 0}(x) d x+\int_{0}^{\infty} \mu_{0}(x) p_{n+1,0}(x) d x+\int_{0}^{\infty} \mu_{1}(x) p_{n+1,1}(x) d x, \quad n \geq 1 .
\end{aligned}
$$

By solving (A.1)-(A.2), we have

$$
\begin{aligned}
p_{0,0} & =\frac{1}{\gamma+\lambda} y_{0} \\
p_{n, j}(x) & =a_{n, j} e^{-\gamma x}+e^{-\gamma x} \int_{0}^{x} y_{n, j}(\tau) e^{\gamma \tau} d \tau, \quad n \geq 1, \quad j=0,1,
\end{aligned}
$$

(A.3)-(A.4) together with (A.5)-(A.6) we get that

$$
\begin{aligned}
a_{1,0}= & p_{1,0}(0)=\lambda p_{0,0}=\frac{\lambda}{\gamma+\lambda} y_{0,0}, \\
a_{n, 0}= & p_{n, 0}(0)=0, \quad n \geq 2, \\
a_{1,1}= & p_{1,1}(0) \\
= & \theta \int_{0}^{\infty} p_{1,0}(x) d x+\int_{0}^{\infty} \mu_{0}(x) p_{2,0}(x) d x+\int_{0}^{\infty} \mu_{1}(x) p_{2,1}(x) d x \\
= & \theta \int_{0}^{\infty}\left[a_{1,0} e^{-\gamma x}+e^{-\gamma x} \int_{0}^{x} y_{1,0}(\tau) e^{\gamma \tau} d \tau\right] d x \\
& +\int_{0}^{\infty} \mu_{0}(x)\left[a_{2,0} e^{-\gamma x}+e^{-\gamma x} \int_{0}^{x} y_{2,0}(\tau) e^{\gamma \tau} d \tau\right] d x \\
& +\int_{0}^{\infty} \mu_{1}(x)\left[a_{2,1} e^{-\gamma x}+e^{-\gamma x} \int_{0}^{x} y_{2,1}(\tau) e^{\gamma \tau} d \tau\right] d x \\
= & \frac{\theta}{\gamma} a_{1,0}+\theta \int_{0}^{\infty} e^{-\gamma x} \int_{0}^{x} y_{1,0}(\tau) e^{\gamma \tau} d \tau d x+\int_{0}^{\infty} \mu_{0}(x) e^{-\gamma x} \int_{0}^{x} y_{2,0}(\tau) e^{\gamma \tau} d \tau d x \\
& +a_{2,1} \int_{0}^{\infty} \mu_{1}(x) e^{-\gamma x} d x+\int_{0}^{\infty} e^{-\gamma x} \int_{0}^{x} y_{2,1}(\tau) e^{\gamma \tau} d \tau d x,
\end{aligned}
$$




$$
\begin{aligned}
a_{n, 1}= & p_{n, 1}(0) \\
= & \theta \int_{0}^{\infty} p_{n, 0}(x) d x+\int_{0}^{\infty} \mu_{0}(x) p_{n+1,0}(x) d x+\int_{0}^{\infty} \mu_{1}(x) p_{n+1,1}(x) d x \\
= & \theta \int_{0}^{\infty}\left[a_{n, 0} e^{-\gamma x}+e^{-\gamma x} \int_{0}^{x} y_{n, 0}(\tau) e^{\gamma \tau} d \tau\right] d x \\
& +\int_{0}^{\infty} \mu_{0}(x)\left[a_{n+1,0} e^{-\gamma x}+e^{-\gamma x} \int_{0}^{x} y_{n+1,0}(\tau) e^{\gamma \tau} d \tau\right] d x \\
& +\int_{0}^{\infty} \mu_{1}(x)\left[a_{n+1,1} e^{-\gamma x}+e^{-\gamma x} \int_{0}^{x} y_{n+1,1}(\tau) e^{\gamma \tau} d \tau\right] d x \\
= & \theta \int_{0}^{\infty} e^{-\gamma x} \int_{0}^{x} y_{n, 0}(\tau) e^{\gamma \tau} d \tau d x+\int_{0}^{\infty} \mu_{0}(x) e^{-\gamma x} \int_{0}^{x} y_{n+1,0}(\tau) e^{\gamma \tau} d \tau d x \\
& +a_{n+1,1} \int_{0}^{\infty} \mu_{1}(x) e^{-\gamma x} d x+\int_{0}^{\infty} \mu_{1}(x) e^{-\gamma x} \int_{0}^{x} y_{n+1,1}(\tau) e^{\gamma \tau} d \tau d x, \quad n \geq 2 .
\end{aligned}
$$

If we set

$$
\begin{aligned}
C & =\left(\begin{array}{ccccc}
1 & -\int_{0}^{\infty} \mu_{1}(x) e^{-\gamma x} d x & 0 & 0 & \cdots \\
0 & 1 & -\int_{0}^{\infty} \mu_{1}(x) e^{-\gamma x} d x & 0 & \ldots \\
0 & 0 & 1 & -\int_{0}^{\infty} \mu_{1}(x) e^{-\gamma x} d x & \ldots \\
\vdots & \vdots & \vdots & \vdots & \ddots
\end{array}\right), \\
\vec{a} & =\left(a_{1,1}, a_{2,1}, a_{3,1}, \cdots\right)^{T},
\end{aligned}
$$

then (A.9)-(A.10) give

$$
\begin{aligned}
& C \vec{a}=\left(\begin{array}{c}
\frac{\theta}{\gamma} a_{1,0}+\theta \int_{0}^{\infty} e^{-\gamma x} \int_{0}^{x} y_{1,0}(\tau) e^{\gamma \tau} d \tau d x+\int_{0}^{\infty} \mu_{0}(x) e^{-\gamma x} \int_{0}^{x} y_{2,0}(\tau) e^{\gamma \tau} d \tau d x \\
\theta \int_{0}^{\infty} e^{-\gamma x} \int_{0}^{x} y_{2,0}(\tau) e^{\gamma \tau} d \tau d x+\int_{0}^{\infty} \mu_{0}(x) e^{-\gamma x} \int_{0}^{x} y_{3,0}(\tau) e^{\gamma \tau} d \tau d x \\
\theta \int_{0}^{\infty} e^{-\gamma x} \int_{0}^{x} y_{3,0}(\tau) e^{\gamma \tau} d \tau d x+\int_{0}^{\infty} \mu_{0}(x) e^{-\gamma x} \int_{0}^{x} y_{4,0}(\tau) e^{\gamma \tau} d \tau d x \\
\theta \int_{0}^{\infty} e^{-\gamma x} \int_{0}^{x} y_{4,0}(\tau) e^{\gamma \tau} d \tau d x+\int_{0}^{\infty} \mu_{0}(x) e^{-\gamma x} \int_{0}^{x} y_{5,0}(\tau) e^{\gamma \tau} d \tau d x \\
\vdots
\end{array}\right. \\
& +\int_{0}^{\infty} \mu_{1}(x) e^{-\gamma x} \int_{0}^{x} y_{2,1}(\tau) e^{\gamma \tau} d \tau d x \\
& +\int_{0}^{\infty} \mu_{1}(x) e^{-\gamma x} \int_{0}^{x} y_{3,1}(\tau) e^{\gamma \tau} d \tau d x \\
& +\int_{0}^{\infty} \mu_{1}(x) e^{-\gamma x} \int_{0}^{x} y_{4,1}(\tau) e^{\gamma \tau} d \tau d x \text {, } \\
& +\int_{0}^{\infty} \mu_{1}(x) e^{-\gamma x} \int_{0}^{x} y_{5,1}(\tau) e^{\gamma \tau} d \tau d x
\end{aligned}
$$

It is easy to calculate the inverse of $C$ as follows:

$$
C^{-1}=\left(\begin{array}{ccccc}
1 & \int_{0}^{\infty} \mu_{1}(x) e^{-\gamma x} d x & \left(\int_{0}^{\infty} \mu_{1}(x) e^{-\gamma x} d x\right)^{2} & \left(\int_{0}^{\infty} \mu_{1}(x) e^{-\gamma x} d x\right)^{3} & \ldots \\
0 & 1 & \int_{0}^{\infty} \mu_{1}(x) e^{-\gamma x} d x & \left(\int_{0}^{\infty} \mu_{1}(x) e^{-\gamma x} d x\right)^{2} & \ldots \\
0 & 0 & 1 & \int_{0}^{\infty} \mu_{1}(x) e^{-\gamma x} d x & \ldots \\
0 & 0 & 0 & 1 & \ldots \\
\vdots & \vdots & \vdots & \vdots & \ddots
\end{array}\right),
$$

which together with (A.11) we obtain that

$$
\begin{aligned}
a_{1,1}= & \frac{\lambda \theta}{\gamma(\gamma+\lambda)} y_{0,0}+\sum_{k=0}^{\infty}\left(\int_{0}^{\infty} \mu_{1}(x) e^{-\gamma x} d x\right)^{k}\left[\theta \int_{0}^{\infty} e^{-\gamma x} \int_{0}^{x} y_{k+1,0}(\tau) e^{\gamma \tau} d \tau d x\right. \\
& \left.+\int_{0}^{\infty} \mu_{0}(x) e^{-\gamma x} \int_{0}^{x} y_{k+2,0}(\tau) e^{\gamma \tau} d \tau d x+\int_{0}^{\infty} \mu_{1}(x) e^{-\gamma x} \int_{0}^{x} y_{k+2,1}(\tau) e^{\gamma \tau} d \tau d x\right], \\
a_{n, 1}= & \sum_{k=0}^{\infty}\left(\int_{0}^{\infty} \mu_{1}(x) e^{-\gamma x} d x\right)^{k}\left[\theta \int_{0}^{\infty} e^{-\gamma x} \int_{0}^{x} y_{k+n, 0}(\tau) e^{\gamma \tau} d \tau d x\right. \\
& \left.+\int_{0}^{\infty} \mu_{0}(x) e^{-\gamma x} \int_{0}^{x} y_{k+n+1,0}(\tau) e^{\gamma \tau} d \tau d x+\int_{0}^{\infty} \mu_{1}(x) e^{-\gamma x} \int_{0}^{x} y_{k+n+1,1}(\tau) e^{\gamma \tau} d \tau d x\right], \quad n \geq 2 .
\end{aligned}
$$


Now, from (A.5)-(A.6) and using the Fubini theorem we calculate (assume $\gamma>0$ )

$$
\begin{aligned}
\left\|p_{n, j}\right\|_{L^{1}[0, \infty)}= & \int_{0}^{\infty}\left|p_{n, j}(x)\right| d x \\
= & \int_{0}^{\infty}\left|a_{n, j} e^{-\gamma x}+e^{-\gamma x} \int_{0}^{x} y_{n, j}(\tau) e^{\gamma \tau} d \tau\right| d x \\
\leq & \left|a_{n, j}\right| \int_{0}^{\infty} e^{-\gamma x} d x+\int_{0}^{\infty} e^{-\gamma x} \int_{0}^{x}\left|y_{n, j}(\tau)\right| e^{\gamma \tau} d \tau d x \\
= & \frac{1}{\gamma}\left|a_{n, j}\right|+\int_{0}^{\infty} y_{n, j}(\tau) e^{\gamma \tau} \int_{\tau}^{\infty} e^{-\gamma x} d x d \tau \\
= & \frac{1}{\gamma}\left|a_{n, j}\right|+\frac{1}{\gamma}\left\|y_{n, j}\right\|_{L^{1}[0, \infty)}, j=0,1, \\
= & \Rightarrow \\
\left\|\left(p_{0}, p_{1}\right)\right\|= & \left\|p_{0}\right\|+\left\|p_{1}\right\| \\
= & \left|p_{0,0}\right|+\sum_{n=1}^{\infty}\left\|p_{n, 0}\right\|_{L^{1}[0, \infty)}+\sum_{n=1}^{\infty}\left\|p_{n, 1}\right\|_{L^{1}[0, \infty)} \\
\leq & \frac{1}{\gamma}\left|y_{0,0}\right|+\frac{1}{\gamma} \sum_{n=1}^{\infty}\left\|y_{n, 0}\right\|_{L^{1}[0, \infty)} \\
& +\frac{1}{\gamma} \sum_{n=1}^{\infty}\left|a_{n, 1}\right|+\frac{1}{\gamma} \sum_{n=1}^{\infty}\left\|y_{n, 1}\right\|_{L^{1}[0, \infty)},
\end{aligned}
$$

Combining (A.12) and (A.13) with the Fubini theorem we estimate

$$
\begin{aligned}
& \sum_{n=1}^{\infty}\left|a_{n, 1}\right| \leq \frac{\lambda \theta}{\gamma(\gamma+\lambda)}\left|y_{0,0}\right| \\
& +\theta \sum_{n=1}^{\infty} \sum_{k=0}^{\infty}\left(\int_{0}^{\infty} \mu_{1}(x) e^{-\gamma x} d x\right)^{k} \int_{0}^{\infty} e^{-\gamma x} \int_{0}^{x}\left|y_{k+n, 0}(\tau)\right| e^{\gamma \tau} d \tau d x \\
& +\sum_{n=1}^{\infty} \sum_{k=0}^{\infty}\left(\int_{0}^{\infty} \mu_{1}(x) e^{-\gamma x} d x\right)^{k} \int_{0}^{\infty} \mu_{0}(x) e^{-\gamma x} \int_{0}^{x}\left|y_{k+n+1,0}(\tau)\right| e^{\gamma \tau} d \tau d x \\
& +\sum_{n=1}^{\infty} \sum_{k=0}^{\infty}\left(\int_{0}^{\infty} \mu_{1}(x) e^{-\gamma x} d x\right)^{k} \int_{0}^{\infty} \mu_{1}(x) e^{-\gamma x} \int_{0}^{x}\left|y_{k+n+1,1}(\tau)\right| e^{\gamma \tau} d \tau d x \\
& =\frac{\lambda \theta}{\gamma(\gamma+\lambda)}\left|y_{0,0}\right| \\
& +\theta \sum_{n=1}^{\infty} \sum_{k=0}^{\infty}\left(\int_{0}^{\infty} \mu_{1}(x) e^{-\gamma x} d x\right)^{k} \int_{0}^{\infty}\left|y_{k+n, 0}(\tau)\right| e^{\gamma \tau} \int_{\tau}^{\infty} e^{-\gamma x} d x d \tau \\
& +\sum_{n=1}^{\infty} \sum_{k=0}^{\infty}\left(\int_{0}^{\infty} \mu_{1}(x) e^{-\gamma x} d x\right)^{k} \int_{0}^{\infty}\left|y_{k+n+1,0}(\tau)\right| e^{\gamma \tau} \int_{\tau}^{\infty} \mu_{0}(x) e^{-\gamma x} d x d \tau \\
& +\sum_{n=1}^{\infty} \sum_{k=0}^{\infty}\left(\int_{0}^{\infty} \mu_{1}(x) e^{-\gamma x} d x\right)^{k} \int_{0}^{\infty}\left|y_{k+n+1,1}(\tau)\right| e^{\gamma \tau} \int_{\tau}^{\infty} \mu_{1}(x) e^{-\gamma x} d x d \tau \\
& \leq \frac{\lambda \theta}{\gamma(\gamma+\lambda)}\left|y_{0,0}\right| \\
& +\theta \sum_{n=1}^{\infty} \sum_{k=0}^{\infty}\left(\int_{0}^{\infty} \overline{\mu_{1}} e^{-\gamma x} d x\right)^{k} \int_{0}^{\infty}\left|y_{k+n, 0}(\tau)\right| e^{\gamma \tau} \int_{\tau}^{\infty} e^{-\gamma x} d x d \tau \\
& +\sum_{n=1}^{\infty} \sum_{k=0}^{\infty}\left(\int_{0}^{\infty} \overline{\mu_{1}} e^{-\gamma x} d x\right)^{k} \int_{0}^{\infty}\left|y_{k+n+1,0}(\tau)\right| e^{\gamma \tau} \int_{\tau}^{\infty} \overline{\mu_{0}} e^{-\gamma x} d x d \tau
\end{aligned}
$$




$$
\begin{aligned}
& +\sum_{n=1}^{\infty} \sum_{k=0}^{\infty}\left(\int_{0}^{\infty} \overline{\mu_{1}} e^{-\gamma x} d x\right)^{k} \int_{0}^{\infty}\left|y_{k+n+1,1}(\tau)\right| e^{\gamma \tau} \int_{\tau}^{\infty} \overline{\mu_{1}} e^{-\gamma x} d x d \tau \\
\leq & \frac{\lambda \theta}{\gamma(\gamma+\lambda)}\left|y_{0,0}\right|+\frac{\theta}{\gamma} \sum_{n=1}^{\infty} \sum_{k=0}^{\infty}\left(\frac{\overline{\mu_{1}}}{\gamma}\right)^{k} \int_{0}^{\infty}\left|y_{k+n, 0}(\tau)\right| d \tau \\
& +\frac{\overline{\mu_{0}}}{\gamma} \sum_{n=1}^{\infty} \sum_{k=0}^{\infty}\left(\frac{\overline{\mu_{1}}}{\gamma}\right)^{k} \int_{0}^{\infty}\left|y_{k+n+1,0}(\tau)\right| d \tau \\
& +\sum_{n=1}^{\infty} \sum_{k=0}^{\infty}\left(\frac{\overline{\mu_{1}}}{\gamma}\right)^{k+1} \int_{0}^{\infty}\left|y_{k+n+1,1}(\tau)\right| d \tau \\
\leq & \frac{\lambda \theta}{\gamma(\gamma+\lambda)}\left|y_{0,0}\right|+\frac{\theta}{\gamma} \sum_{k=0}^{\infty}\left(\frac{\overline{\mu_{1}}}{\gamma}\right)^{k} \sum_{n=1}^{\infty}\left\|y_{n, 0}\right\|_{L^{1}[0, \infty)} \\
+ & \frac{\overline{\mu_{0}}}{\gamma} \sum_{k=0}^{\infty}\left(\frac{\overline{\mu_{1}}}{\gamma}\right)^{k} \sum_{n=1}^{\infty}\left\|y_{n, 0}\right\|_{L^{1}[0, \infty)}+\sum_{k=0}^{\infty}\left(\frac{\overline{\mu_{1}}}{\gamma}\right)^{k+1} \sum_{n=1}^{\infty}\left\|y_{n, 1}\right\|_{L^{1}[0, \infty)} \\
& =\frac{\lambda \theta}{\gamma(\gamma+\lambda)}\left|y_{0,0}\right|+\frac{\theta}{\gamma} \frac{\gamma}{\gamma-\overline{\mu_{1}}} \sum_{n=1}^{\infty}\left\|y_{n, 0}\right\|_{L^{1}[0, \infty)} \\
& +\frac{\overline{\mu_{0}}}{\gamma} \frac{\gamma}{\gamma-\overline{\mu_{1}}} \sum_{n=1}^{\infty}\left\|y_{n, 0}\right\|_{L^{1}[0, \infty)}+\frac{\overline{\mu_{1}}}{\gamma-\overline{\mu_{1}}} \sum_{n=1}^{\infty}\left\|y_{n, 1}\right\|_{L^{1}[0, \infty)} \\
& =\frac{\lambda \theta}{\gamma(\gamma+\lambda)}\left|y_{0,0}\right|+\frac{\overline{\mu_{0}}+\theta}{\gamma-\overline{\mu_{1}}} \sum_{n=1}^{\infty}\left\|y_{n, 0}\right\|_{L^{1}[0, \infty)} \\
& +\frac{\overline{\mu_{1}}}{\gamma-\overline{\mu_{1}}} \sum_{n=1}^{\infty}\left\|y_{n, 1}\right\|_{L^{1}[0, \infty)} .
\end{aligned}
$$

By substituting the (A.15) into (A.14) we estimate (assume $\overline{\mu_{1}}>\overline{\mu_{0}}+\theta$ and $\gamma>\overline{\mu_{1}}$ )

$$
\begin{aligned}
\left\|\left(p_{0}, p_{1}\right)\right\| \leq & \frac{1}{\gamma}\left|y_{0,0}\right|+\frac{1}{\gamma} \sum_{n=1}^{\infty}\left\|y_{n, 0}\right\|_{L^{1}[0, \infty)} \\
& +\frac{1}{\gamma}\left\{\frac{\lambda \theta}{\gamma(\gamma+\lambda)}\left|y_{0,0}\right|+\frac{\overline{\mu_{0}}+\theta}{\gamma-\overline{\mu_{1}}} \sum_{n=1}^{\infty}\left\|y_{n, 0}\right\|_{L^{1}[0, \infty)}\right. \\
& \left.+\frac{\overline{\mu_{1}}}{\gamma-\overline{\mu_{1}}} \sum_{n=1}^{\infty}\left\|y_{n, 1}\right\|_{L^{1}[0, \infty)}\right\}+\frac{1}{\gamma} \sum_{n=1}^{\infty}\left\|y_{n, 1}\right\|_{L^{1}[0, \infty)} \\
\leq & \frac{\gamma^{2}+\lambda \gamma+\lambda \theta}{\gamma^{2}(\gamma+\lambda)}\left|y_{0,0}\right|+\frac{\gamma-\overline{\mu_{1}}+\overline{\mu_{0}}+\theta}{\gamma\left(\gamma-\overline{\mu_{1}}\right)} \sum_{n=1}^{\infty}\left\|y_{n, 0}\right\|_{L^{1}[0, \infty)} \\
& +\frac{1}{\gamma-\overline{\mu_{1}}} \sum_{n=1}^{\infty}\left\|y_{n, 1}\right\|_{L^{1}[0, \infty)} \\
\leq & \frac{1}{\gamma-\overline{\mu_{1}}}\left\{\left|y_{0,0}\right|+\sum_{n=1}^{\infty}\left\|y_{n, 0}\right\|_{L^{1}[0, \infty)}+\sum_{n=1}^{\infty}\left\|y_{n, 1}\right\|_{L^{1}[0, \infty)}\right\} \\
= & \frac{1}{\gamma-\overline{\mu_{1}}}\|(y, z)\| .
\end{aligned}
$$

(A.16) shows that $(\gamma I-A)^{-1}$ exist for $\gamma>\overline{\mu_{1}}$, and

$$
(\gamma I-A)^{-1}: X \rightarrow D(A), \quad\left\|(\gamma I-A)^{-1}\right\| \leq \frac{1}{\gamma-\overline{\mu_{1}}} .
$$

In the following, we will prove that $D(A)$ is dense in $X$. Since $\forall\left(p_{0}, p_{1}\right) \in X$ implies

$$
\left|p_{0,0}\right|+\sum_{n=1}^{\infty}\left\|p_{n, 0}\right\|_{L^{1}[0, \infty)}+\sum_{n=1}^{\infty}\left\|p_{n, 1}\right\|_{L^{1}[0, \infty)}<\infty
$$


It follows that, for any $\epsilon>0$, there exists a positive integer $K$ such that such that

$$
\sum_{n=K}^{\infty}\left\|p_{n, 0}\right\|_{L^{1}[0, \infty)}+\sum_{n=K}^{\infty}\left\|p_{n, 1}\right\|_{L^{1}[0, \infty)}<\epsilon
$$

Which shows that the set

$$
L=\left\{\begin{array}{l|l}
\left(p_{0}, p_{1}\right) & \begin{array}{l}
p_{0}(x)=\left(p_{0,0}, p_{1,0}(x), p_{2,0}(x), \cdots, p_{K, 0}(x), 0,0, \cdots\right) \\
p_{1}(x)=\left(p_{1,1}(x), p_{2,1}(x), \cdots, p_{K, 1}(x), 0,0, \cdots\right) \\
p_{i, 0}(x), p_{i, 1}(x) \in L^{1}[0, \infty), \quad i=1,2, \cdots, K \\
K \text { is a finite positive integer. }
\end{array}
\end{array}\right\}
$$

is dense in $X$. If we set

$$
Z=\left\{\begin{array}{l|l}
\left(p_{0}, p_{1}\right) & \begin{array}{l}
p_{0}(x)=\left(p_{0,0}, p_{1,0}(x), p_{2,0}(x), \cdots, p_{N, 0}(x), 0,0, \cdots\right) \\
p_{1}(x)=\left(p_{1,1}(x), p_{2,1}(x), \cdots, p_{N, 1}(x), 0,0, \cdots\right) \\
p_{i, 0}(x), p_{i, 1}(x) \in C_{0}^{\infty}[0, \infty), \text { there exists } c_{i}>0, d_{i}>0 \\
\text { such that } p_{i, 0}(x)=0, x \in\left[0, c_{i}\right] ; p_{i, 1}(x)=0, x \in\left[0, d_{i}\right] \\
i=1,2, \cdots, N .
\end{array}
\end{array}\right\}
$$

then from (Adams, 1975) we know that $Z$ is dense in $L$. Therefore, in order to prove that $D(A)$ is dense in $X$, it is suffices to prove $Z \subset \overline{D(A)}$. In fact, if $Z \subset \overline{D(A)}$, then $X=\bar{L}=\overline{\bar{Z}}=\bar{Z} \subset \overline{\overline{D(A)}}=\overline{D(A)} \subset X$ implies $X=\overline{D(A)}$.

Take any $\left(p_{0}, p_{1}\right) \in Z$, there are a finite positive integer $N$ and positive numbers $c_{i}>0, d_{i}>0$ such that, for $i=1,2, \cdots N$

$$
\begin{aligned}
& p_{0}(x)=\left(p_{0,0}, p_{1,0}(x), p_{2,0}(x), \cdots, p_{N, 0}(x), 0,0, \cdots\right), p_{i, 0}(x)=0, x \in\left[0, c_{i}\right] \\
& p_{1}(x)=\left(p_{1,1}(x), p_{2,1}(x), \cdots, p_{N, 1}(x), 0,0, \cdots\right), p_{i, 1}(x)=0, x \in\left[0, d_{i}\right]
\end{aligned}
$$

This implies $p_{i, 0}(x)=0, p_{i, 1}(x)=0$ for $x \in[0,2 s]$ where $0<2 s<\min \left\{c_{0}, c_{1}, \cdots, c_{N}, d_{0}, d_{1}, \cdots d_{N}\right\}$. Define

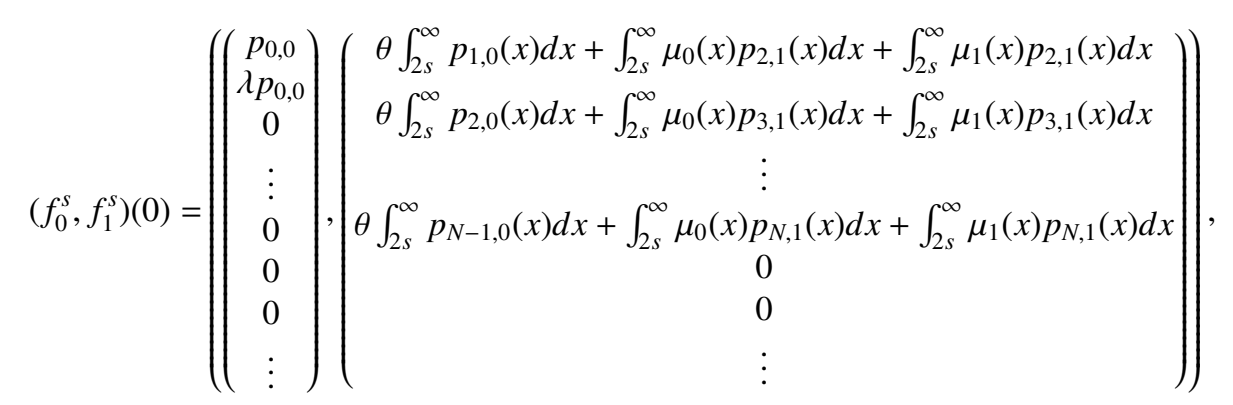

$$
\left(f_{0}^{s}, f_{1}^{s}\right)(x)=\left(\left(\begin{array}{c}
p_{0,0} \\
f_{1,0}^{s}(x) \\
f_{2,0}^{s}(x) \\
\vdots \\
f_{N-1,0}^{s}(x) \\
f_{N, 0}^{s}(x) \\
0 \\
0 \\
\vdots
\end{array}\right),\left(\begin{array}{c}
f_{1,1}^{s}(x) \\
f_{2,1}^{s}(x) \\
f_{3,1}^{s}(x) \\
\vdots \\
f_{N-1,1}^{s}(x) \\
f_{N, 1}^{s}(x) \\
0 \\
0 \\
\vdots
\end{array}\right)\right)
$$


here

$$
\begin{aligned}
& f_{i, 0}^{s}(x)=\left\{\begin{array}{ll}
f_{i, 0}^{s}(0)\left(1-\frac{x}{s}\right)^{2} & x \in[0, s), \\
-u_{i}(x-s)^{2}(x-2 s)^{2} & x \in[s, 2 s), \\
p_{i, 0}(x) & x \in[2 s, \infty) .
\end{array} \quad f_{N, 0}^{s}(x)=p_{N, 0}(x),\right. \\
& u_{1}=\frac{\int_{0}^{s} f_{i, 0}^{s}(0)\left(1-\frac{x}{s}\right)^{2} d x}{\int_{s}^{2 s}(x-s)^{2}(x-2 s)^{2} d x} \\
& u_{i}=\frac{\int_{0}^{s} f_{i, 0}^{s}(0)\left(1-\frac{x}{s}\right)^{2} \mu_{0}(x) d x}{\int_{s}^{2 s}(x-s)^{2}(x-2 s)^{2} \mu_{0}(x) d x}, \quad i=2,3, \cdots, N \\
& f_{i, 1}^{s}(x)=\left\{\begin{array}{ll}
f_{i, 1}^{s}(0)\left(1-\frac{x}{s}\right)^{2} & x \in[0, s), \\
-v_{i}(x-s)^{2}(x-2 s)^{2} & x \in[s, 2 s), \\
p_{i, 1}(x) & x \in[2 s, \infty) .
\end{array} \quad f_{N, 1}^{s}(x)=p_{N, 1}(x),\right. \\
& v_{i}=\frac{1}{\int_{s}^{2 s}(x-s)^{2}(x-2 s)^{2} \mu_{1}(x) d x}\left\{\int_{0}^{s} f_{i, 1}^{s}(0)\left(1-\frac{x}{s}\right)^{2} \mu_{1}(x) d x\right. \\
& +\int_{0}^{s} f_{i-1,0}^{s}(0)\left(1-\frac{x}{s}\right)^{2} \mu_{0}(x) d x-\theta u_{i-1} \int_{s}^{2 s}(x-s)^{2}(x-2 s)^{2} \mu_{0}(x) d x \\
& \left.+\int_{0}^{s} f_{i-1,0}^{s}(0)\left(1-\frac{x}{s}\right)^{2} d x-\theta u_{i-1} \int_{s}^{2 s}(x-s)^{2}(x-2 s)^{2} d x\right\}, \quad i=2,3 \cdots, N .
\end{aligned}
$$

It is easy to verify that $\left(f_{0}^{s}, f_{1}^{s}\right) \in D(A)$. Moreover

$$
\begin{aligned}
\left\|\left(p_{0}, p_{1}\right)-\left(f_{0}^{s}, f_{1}^{s}\right)\right\|= & \sum_{n=1}^{N} \int_{0}^{\infty}\left|p_{n, 0}(x)-f_{n, 0}^{s}(x)\right| d x+\sum_{n=2}^{N} \int_{0}^{\infty}\left|p_{n, 1}(x)-f_{n, 1}^{s}(x)\right| d x \\
= & \sum_{n=1}^{N} \int_{0}^{s}\left|f_{n, 0}^{s}(0)\right|\left(1-\frac{x}{s}\right)^{2} d x+\sum_{n=1}^{N} \int_{s}^{2 s}\left|u_{n}\right|(x-s)^{2}(x-2 s)^{2} d x \\
& +\sum_{n=2}^{N} \int_{0}^{s}\left|f_{n, 1}^{s}(0)\right|\left(1-\frac{x}{s}\right)^{2} d x+\sum_{n=2}^{N} \int_{s}^{2 s}\left|v_{n}\right|(x-s)^{2}(x-2 s)^{2} d x \\
= & \sum_{n=1}^{N}\left|f_{n, 0}^{s}(0)\right| \frac{s}{3}+\sum_{n=1}^{N}\left|u_{n}\right| \frac{s^{5}}{30}+\sum_{n=2}^{N}\left|f_{n, 1}^{s}(0)\right| \frac{s}{3}+\sum_{n=2}^{N}\left|v_{n}\right| \frac{s^{5}}{30} \\
\rightarrow & 0, \quad \text { as } s \rightarrow 0 .
\end{aligned}
$$

This means that $D(A)$ is dense in $X$. From the above two steps and Hille-Yosida Theorem we conclude that $A$ generates a $C_{0}-$ semigroup. see (Gupur, $\mathrm{Li}, \& \mathrm{Zhu}, 2001$ ). Now, we verify that $U$ and $E$ are bounded linear operators. From the definition of $U$ and $E$ we have, for $\left(p_{0}, p_{1}\right) \in X$

$$
\begin{aligned}
\left\|U\left(p_{0}, p_{1}\right)\right\| \leq & \sum_{n=1}^{\infty} \int_{0}^{\infty}\left|\left(\lambda+\theta+\mu_{0}(x)\right) p_{n, 0}(x)\right| d x+\sum_{n=2}^{\infty} \int_{0}^{\infty}\left|\lambda p_{n, 0}(x)\right| d x \\
& +\sum_{n=1}^{\infty} \int_{0}^{\infty}\left|\left(\lambda+\mu_{1}(x)\right) p_{n, 1}(x)\right| d x+\sum_{n=2}^{\infty} \int_{0}^{\infty}\left|\lambda p_{n, 1}(x)\right| d x \\
\leq & \left(2 \lambda+\theta+\overline{\mu_{0}}\right) \sum_{n=1}^{\infty} \int_{0}^{\infty}\left|p_{n, 0}(x)\right| d x+\left(2 \lambda+\overline{\mu_{1}}\right) \sum_{n=1}^{\infty} \int_{0}^{\infty}\left|p_{n, 1}(x)\right| d x \\
\leq & \max \left\{2 \lambda+\theta+\overline{\mu_{0}}, 2 \lambda+\overline{\mu_{1}}\right\}\left\|\left(p_{0}, p_{1}\right)\right\|, \\
\left\|E\left(p_{0}, p_{1}\right)\right\| \leq & \overline{\mu_{0}} \int_{0}^{\infty}\left|p_{1,0}(x)\right| d x+\overline{\mu_{1}} \int_{0}^{\infty}\left|p_{1,1}(x)\right| d x \\
\leq & \max \left\{\overline{\mu_{0}}, \overline{\mu_{1}}\right\}\left\|\left(p_{0}, p_{1}\right)\right\| .
\end{aligned}
$$

(A.17) and (A.18) show that $U$ and $E$ are bounded linear operators. It is easy to see that $U$ and $E$ are linear operators. Hence, From the perturbation theory of $C_{0}$-semigroup we get that $A+U+E$ generates a $C_{0}-\operatorname{semigroup~} T(t)$. 
Finally, we prove that $A+U+E$ is dispersive operator. For $\left(p_{0}, p_{1}\right) \in D(A)$, we choose

$$
\phi_{j}(x)=\left(\frac{\left[p_{0, j}\right]^{+}}{p_{0, j}}, \frac{\left[p_{1, j}(x)\right]^{+}}{p_{1, j}(x)}, \frac{\left[p_{2, j}(x)\right]^{+}}{p_{2, j}(x)}, \cdots\right), \quad j=0,1 .
$$

Where

$$
\left[p_{0,0}\right]^{+}=\left\{\begin{array}{lll}
p_{0,0} & \text { if } & p_{0,0}>0 \\
0 & \text { if } & p_{0,0} \leq 0
\end{array}, \quad\left[p_{n, j}(x)\right]^{+}=\left\{\begin{array}{lll}
p_{n, j}(x) & \text { if } & p_{n, j}(x)>0 \\
0 & \text { if } & p_{n, j}(x) \leq 0
\end{array}, \quad n \geq 1, j=0,1 .\right.\right.
$$

The boundary condition on $\left(p_{0}, p_{1}\right) \in D(A)$ imply

$$
\begin{aligned}
\sum_{n=1}^{\infty}\left[p_{n, 0}(0)\right]^{+} \leq & \lambda\left[p_{0,0}\right]^{+} \\
\sum_{n=1}^{\infty}\left[p_{n, 1}(0)\right]^{+} \leq & \theta \sum_{n=1}^{\infty} \int_{0}^{\infty}\left[p_{n, 0}(x)\right]^{+} d x+\sum_{n=1}^{\infty} \int_{0}^{\infty} \mu_{0}(x)\left[p_{n+1,0}(x)\right]^{+} d x \\
& +\sum_{n=1}^{\infty} \int_{0}^{\infty} \mu_{1}(x)\left[p_{n+1,1}(x)\right]^{+} d x
\end{aligned}
$$

If we define $V_{i, j}=\left\{x \in[0, \infty) \mid p_{i, j}(x)>0\right\}$ and $W_{i, j}=\left\{x \in[0, \infty) \mid p_{i, j}(x) \leq 0\right\}$ for $i \geq 1, j=0,1$, then we have

$$
\begin{aligned}
\int_{0}^{\infty} \frac{d p_{i, j}(x)}{d x} \frac{\left[p_{i, j}(x)\right]^{+}}{p_{i, j}(x)} d x & =\int_{V_{i, j}} \frac{d p_{i, j}(x)}{d x} \frac{\left[p_{i, j}(x)\right]^{+}}{p_{i, j}(x)} d x+\int_{W_{i, j}} \frac{d p_{i, j}(x)}{d x} \frac{\left[p_{i, j}(x)\right]^{+}}{p_{i, j}(x)} d x \\
& =\int_{V_{i, j}} \frac{d p_{i, j}(x)}{d x} \frac{\left[p_{i, j}(x)\right]^{+}}{p_{i, j}(x)} d x=\int_{V_{i, j}} \frac{d p_{i, j}(x)}{d x} d x \\
& =\int_{V_{i, j}} \frac{d\left[p_{i, j}(x)\right]^{+}}{d x} d x=-\left[p_{i, j}(0)\right]^{+}, \quad i \geq 1, j=0,1 .
\end{aligned}
$$

By using boundary condition on $\left(p_{0}, p_{1}\right) \in D(A)$ and (A.19)-(A.21) for such $\left(\phi_{0}, \phi_{1}\right)$, we derive

$$
\begin{aligned}
\langle(A & \left.+U+E)\left(p_{0}, p_{1}\right),\left(\phi_{0}, \phi_{1}\right)\right\rangle \\
= & \left.-\lambda p_{0,0}+\int_{0}^{\infty} \mu_{0}(x) p_{1,0}(x) d x+\int_{0}^{\infty} \mu_{1}(x) p_{1,1}(x) d x\right\} \frac{\left[p_{0,0}\right]^{+}}{p_{0,0}} \\
& +\int_{0}^{\infty}\left\{-\frac{d p_{1,0}(x)}{d x}-\left(\lambda+\theta+\mu_{0}(x)\right) p_{1,0}(x)\right\} \frac{\left[p_{1,0}(x)\right]^{+}}{p_{1,0}(x)} d x \\
& +\sum_{n=2}^{\infty} \int_{0}^{\infty}\left\{-\frac{d p_{n, 0}(x)}{d x}-\left(\lambda+\theta+\mu_{0}(x)\right) p_{n, 0}(x)+\lambda p_{n-1,0}(x)\right\} \frac{\left[p_{n, 0}(x)\right]^{+}}{p_{n, 0}(x)} d x \\
& +\int_{0}^{\infty}\left\{-\frac{d p_{1,1}(x)}{d x}-\left(\lambda+\mu_{1}(x)\right) p_{1,1}(x)\right\} \frac{\left[p_{1,1}(x)\right]^{+}}{p_{1,1}(x)} d x \\
& +\sum_{n=2}^{\infty} \int_{0}^{\infty}\left\{-\frac{d p_{n, 1}(x)}{d x}-\left(\lambda+\mu_{1}(x)\right) p_{n, 1}(x)+\lambda p_{n-1,1}(x)\right\} \frac{\left[p_{n, 1}(x)\right]^{+}}{p_{n, 1}(x)} d x \\
= & -\lambda\left[p_{0,0}\right]^{+}+\left\{\int_{0}^{\infty} \mu_{0}(x) p_{1,0}(x) d x+\int_{0}^{\infty} \mu_{1}(x) p_{1,1}(x) d x\right\} \frac{\left[p_{0,0}\right]^{+}}{p_{0,0}} \\
& -\sum_{n=1}^{\infty} \int_{0}^{\infty} \frac{d p_{n, 0}(x)}{d x} \frac{\left[p_{n, 0}(x)\right]^{+}}{p_{n, 0}(x)} d x-\sum_{n=1}^{\infty} \int_{0}^{\infty}\left(\lambda+\theta+\mu_{0}(x)\right)\left[p_{n, 0}(x)\right]^{+} d x \\
& +\lambda \sum_{n=2}^{\infty} \int_{0}^{\infty} p_{n-1,0}(x) \frac{\left[p_{n, 0}(x)\right]^{+}}{p_{n, 0}(x)} d x-\sum_{n=1}^{\infty} \int_{0}^{\infty} \frac{d p_{n, 1}(x)}{d x} \frac{\left[p_{n, 1}(x)\right]^{+}}{p_{n, 1}(x)} d x \\
& -\sum_{n=1}^{\infty} \int_{0}^{\infty}\left(\lambda+\mu_{1}(x)\right)\left[p_{n, 1}(x)\right]^{+} d x+\lambda \sum_{n=2}^{\infty} \int_{0}^{\infty} p_{n-1,1}(x) \frac{\left[p_{n, 1}(x)\right]^{+}}{p_{n, 1}(x)} d x
\end{aligned}
$$




$$
\begin{aligned}
& =-\lambda\left[p_{0,0}\right]^{+}+\left\{\int_{0}^{\infty} \mu_{0}(x) p_{1,0}(x) d x+\int_{0}^{\infty} \mu_{1}(x) p_{1,1}(x) d x\right\} \frac{\left[p_{0,0}\right]^{+}}{p_{0,0}} \\
& +\sum_{n=1}^{\infty}\left[p_{n, 0}(0)\right]^{+}-\sum_{n=1}^{\infty} \int_{0}^{\infty}\left(\lambda+\theta+\mu_{0}(x)\right)\left[p_{n, 0}(x)\right]^{+} d x \\
& +\lambda \sum_{n=2}^{\infty} \int_{0}^{\infty} p_{n-1,0}(x) \frac{\left[p_{n, 0}(x)\right]^{+}}{p_{n, 0}(x)} d x+\sum_{n=1}^{\infty}\left[p_{n, 1}(0)\right]^{+} \\
& -\sum_{n=1}^{\infty} \int_{0}^{\infty}\left(\lambda+\mu_{1}(x)\right)\left[p_{n, 1}(x)\right]^{+} d x+\lambda \sum_{n=2}^{\infty} \int_{0}^{\infty} p_{n-1,1}(x) \frac{\left[p_{n, 1}(x)\right]^{+}}{p_{n, 1}(x)} d x \\
& \leq-\lambda\left[p_{0,0}\right]^{+}+\left\{\int_{0}^{\infty} \mu_{0}(x)\left[p_{1,0}(x)\right]^{+} d x+\int_{0}^{\infty} \mu_{1}(x)\left[p_{1,1}(x)\right]^{+} d x\right\} \frac{\left[p_{0,0}\right]^{+}}{p_{0,0}} \\
& +\lambda\left[p_{0,0}\right]^{+}-\sum_{n=1}^{\infty} \int_{0}^{\infty}\left[\lambda+\theta+\mu_{0}(x)\right]\left[p_{n, 0}(x)\right]^{+} d x \\
& +\lambda \sum_{n=2}^{\infty} \int_{0}^{\infty}\left[p_{n-1,0}(x)\right]^{+} \frac{\left[p_{n, 0}(x)\right]^{+}}{p_{n, 0}(x)} d x+\theta \sum_{n=1}^{\infty} \int_{0}^{\infty}\left[p_{n, 0}(x)\right]^{+} d x \\
& +\sum_{n=1}^{\infty} \int_{0}^{\infty} \mu_{0}(x)\left[p_{n+1,0}(x)\right]^{+} d x+\sum_{n=1}^{\infty} \int_{0}^{\infty} \mu_{1}(x)\left[p_{n+1,1}(x)\right]^{+} d x \\
& -\sum_{n=1}^{\infty} \int_{0}^{\infty}\left(\lambda+\mu_{1}(x)\right)\left[p_{n, 1}(x)\right]^{+} d x+\lambda \sum_{n=2}^{\infty} \int_{0}^{\infty}\left[p_{n-1,1}(x)\right]^{+} \frac{\left[p_{n, 1}(x)\right]^{+}}{p_{n, 1}(x)} d x \\
& =\left\{\int_{0}^{\infty} \mu_{0}(x)\left[p_{1,0}(x)\right]^{+} d x+\int_{0}^{\infty} \mu_{1}(x)\left[p_{1,1}(x)\right]^{+} d x\right\} \frac{\left[p_{0,0}\right]^{+}}{p_{0,0}} \\
& -\int_{0}^{\infty} \mu_{0}(x)\left[p_{1,0}(x)\right]^{+} d x-\lambda \sum_{n=1}^{\infty} \int_{0}^{\infty}\left[p_{n, 0}(x)\right]^{+} d x \\
& +\lambda \sum_{n=2}^{\infty} \int_{0}^{\infty}\left[p_{n-1,0}(x)\right]^{+} \frac{\left[p_{n, 0}(x)\right]^{+}}{p_{n, 0}(x)} d x-\int_{0}^{\infty} \mu_{1}(x)\left[p_{1,1}(x)\right]^{+} d x \\
& -\lambda \sum_{n=1}^{\infty} \int_{0}^{\infty}\left[p_{n, 1}(x)\right]^{+} d x+\lambda \sum_{n=2}^{\infty} \int_{0}^{\infty}\left[p_{n-1,1}(x)\right]^{+} \frac{\left[p_{n, 1}(x)\right]^{+}}{p_{n, 1}(x)} d x \\
& \leq\left(\frac{\left[p_{0,0}\right]^{+}}{p_{0,0}}-1\right) \int_{0}^{\infty} \mu_{0}(x)\left[p_{1,0}(x)\right]^{+} d x \\
& +\frac{\left[p_{0,0}\right]^{+}}{p_{0,0}} \int_{0}^{\infty} \mu_{1}(x)\left[p_{1,1}(x)\right]^{+} d x \\
& \leq 0 \text {. }
\end{aligned}
$$

In the above, we have used the following inequalities:

$$
\begin{aligned}
\int_{0}^{\infty} p_{n-1, j}(x) \frac{\left[p_{n, j}(x)\right]^{+}}{p_{n, j}(x)} d x & \leq \int_{0}^{\infty}\left[p_{n-1, j}(x)\right]^{+} \frac{\left[p_{n, j}(x)\right]^{+}}{p_{n, j}(x)} d x \\
& \leq \int_{0}^{\infty}\left[p_{n-1, j}(x)\right]^{+} d x, \quad n \geq 1, j=0,1 .
\end{aligned}
$$

(A.22) shows that $A+U+E$ is a dispersive operator.

From the first step, the second step, the fourth step and Fillips theorem we obtained that $A+U+E$ generates a positive contraction $C_{0}-$ semigroup. By the uniqueness theorem of the semigroup it follows that this positive contraction $C_{0}$-semigroup is just $T(t)$. Thus, we complete the Theorem 2.1 .

\section{References}

Adams, R. A. (1975). Sobolev spaces. Academic Press, New York.

Doshi, B. T. (1986). Queueing systems with vacations - a survey. Queueing systems, 1(1), 29-66.

http://dx.doi.org/10.1007/BF01149327 
Fattorini, H. O. (1983). The Cauchy Problem. Addison-Wesley, Massachusetts.

Gupur, G. (2002). Well-posedness of M/G/1 queueing model with single vacations. Computers \& Mathematics with Applications, 44(8), 1041-1056. http://dx.doi.org/10.1016/S0898-1221(02)00213-4

Gupur, G. (2010). On the M/M/1 queueing model with compulsory server vacations. International journal of pure and applied mathematics, 64(2), 253-304.

Gupur, G. (2011). Functional analysis methods for reliability models. Springer, Basel.

Gupur, G. (2014). On eigenvalues of the generator of a $C_{0}$-semigroup appearing in queueing theory. Abstract and Applied Analysis, Art. ID 896342, 9 pp. http://dx.doi.org/10.1155/2014/896342

Gupur, G., \& Guo, B. Z. (2002). Asymptotic property of the solution of exhaustive-service M/M/1 queueing model with single vacations. International Journal of Differential Equations and Applications, 1, 29-51.

Gupur, G., Li, X. Z., \& Zhu, G. T. (2001). Functional Analysis Method in Queueing Theory. Research Information Ltd., Hertfordshire.

Jain, M., \& Agrawal, P. K. (2007). $M / E^{k} / 1$ queueing system with working vacation. Quality Technology \& Quantitative Management, 4(4), 455-470. http://dx.doi.org/10.1080/16843703.2007.11673165

Kim, J. D. Choi, D. W., \& Chae, K. C. (2003) Analysis of queue-length distribution of the M/G/l queue with working vacations. in: Hawaii International Conference on Statistics and Related Fields.

Kasim, E., \& Gupur, G. (2011). Other eigenvalues of the M/M/1 operator. Acta Analysis Functionalis Applicata, 13, 45-53.

Li, J. H., Tian, N. S., \& Ma, Z. Y. (2007). Performance analysis of GI/M/1 queue with working vacations and vacation interruption. Applied Mathematical Modelling, 31, 880-894. http://dx.doi.org/10.1016/j.apm.2007.09.017

Lu, Z. J., \& Gupur, G. (2010). Well-posedness of M/G/1 queueing model with compulsory server vacations. Mathematics in Theory and Practice, 40(5), 139-148.

Madan, K. C. (1992). An M/G/1 queueing system with compulsory server vacations. Trabajos de investigación operativa, 7(1), 105-115. http://dx.doi.org/10.1007/BF02888261

Servi, L. D., \& Finn, S. G. (2002). M/M/1 queues with working vacations (M/M/1/WV). Perform. Eval., 50(1), 41-52. http://dx.doi.org/10.1016/S0166-5316(02)00057-3

Takagi, H. (1990). Time-dependent analysis of M/G/1 vacation models with exhaustive service. Queueing Systems, 6, 369-389. http://dx.doi.org/10.1007/BF02411484

Takagi, H., \& Wu, D. (2006). M/G/1 queue with multiple working vacations. Perform. Eval., 63, 654-681. http://dx.doi.org/10.1016/j.peva.2005.05.005

Zhang, M., \& Hou, Z. (2010). Performance analysis of M/G/1 queue with working vacation and vacation interruption. Journal of Computational and Applied Mathematics, 234(10), 2977-2985.

http://dx.doi.org/10.1016/j.cam.2010.04.010

\section{Copyrights}

Copyright for this article is retained by the author(s), with first publication rights granted to the journal.

This is an open-access article distributed under the terms and conditions of the Creative Commons Attribution license (http://creativecommons.org/licenses/by/4.0/). 